\title{
A novel class of carbonic anhydrase inhibitors: glycoconjugate benzene sulfonamides prepared by "click-tailing"
}

Brendan L. Wilkinson,${ }^{\dagger}$ Laurent F. Bornaghi, ${ }^{\dagger}$ Todd A. Houston, $₫$ Alessio Innocenti, $§$ Claudiu T. Supuran ${ }^{*} \S$ and Sally-Ann Poulsen ${ }^{*, \dagger}$

Eskitis Institute for Cell and Molecular Therapies, Griffith University, 170 Kessels Road, Nathan, Queensland 4111, Australia, Institute for Glycomics, Griffith University, PMB 50 Gold Coast Mail Centre, Gold Coast, Queensland 9726, Australia, and Polo Scientifico, Laboratorio di Chimica Bioinorganica, Rm. 188, Università degli Studi di Firenze, Via della Lastruccia 3, 50019 Sesto Fiorentino, Florence, Italy

*Corresponding authors. S.-A.P. Telephone: +61 73735 7825; Fax: +61 73735 7656; e-mail: s.poulsen@griffith.edu.au. C.T.S. Telephone: +39 055457 3005; Fax: +39 055457 3385; email: claudiu.supuran@unifi.it.

$\dagger, \ddagger$ Griffith University

$\S$ Università degli Studi di Firenze

$¥$ Nonstandard abbreviations: CA, carbonic anhydrase; 1,3-DCR, 1,3-dipolar cycloaddition reaction; HBTU, O-benzotriazol-1-yl- $N, N, N^{\prime}, N^{\prime}$-tetramethyluronium hexafluorophosphate; AZA, acetazolamide; MZA, methazolamide; EZA, ethoxzolamide; DCP, dichlorophenamide; BRZ, brinzolamide; IND, Indisulam 


\begin{abstract}
Aryl and heteroaryl sulfonamides $\left(\mathrm{ArSO}_{2} \mathrm{NH}_{2}\right)$ are therapeutically used to inhibit the catalytic activity of carbonic anhydrases (CAs). Using a 'click-tail' approach a novel class of glycoconjugate benzene sulfonamides have been synthesized that contain diverse carbohydrate-triazole tails. These compounds were assessed for their ability to inhibit three human CA isozymes in vitro: cytosolic hCA I and hCA II, and transmembrane, tumourassociated hCA IX. This isozyme has a minimal expression in normal tissue but is overexpressed in hypoxic tumours and its inhibition is a current approach towards new cancer therapies. The qualitative structure-activity for all derivatives demonstrated that the stereochemical diversity present within the carbohydrate tails effectively interrogated the CA active site topology, to generate several inhibitors that were potent and selective towards hCA IX - an important outcome in the quest for potential cancer therapy applications based on CA inhibition.
\end{abstract}




\section{Introduction}

The carbonic anhydrase (CA, EC 4.2.1.1) family of $\mathrm{Zn}(\mathrm{II})$ metalloenzymes is ubiquitous to all eukaryotic and most prokaryotic cells. ${ }^{1}$ This enzyme efficiently catalyses the hydration of carbon dioxide $\left(\mathrm{CO}_{2}\right)$ to give bicarbonate anion $\left(\mathrm{HCO}_{3}^{-}\right)$and a proton $\left(\mathrm{H}^{+}\right)$, a regulatory reaction that underpins fundamental physiological processes associated with $\mathrm{pH}$ control, ion transport and fluid secretion. ${ }^{2-4}$ For decades, inhibitors of CA have been a mainstay of human clinical intervention for a range of diseases, however more recently a role for CA inhibition as an anticancer therapy has been identified owing to the overexpression of some CA isoforms (CA IX and CA XII) in cancer cells and a minimal expression in normal tissue. The latter has further increased interest in this enzyme family as a therapeutic target. ${ }^{5-7}$ The classical recognition fragment for small molecules to bind the active site of $\mathrm{CA}$ is an aromatic sulfonamide moiety - $\mathrm{ArSO}_{2} \mathrm{NH}_{2} \cdot{ }^{1-3,8}$ The sulfonamide anion $\left(\mathrm{ArSO}_{2} \mathrm{NH}^{-}\right)$coordinates to the CA active site $\mathrm{Zn}(\mathrm{II})$ and so inhibits the catalytic ability of the enzyme. This aromatic sulfonamide group has served as a very reliable anchor upon which medicinal chemists have appended 'tails' to deliver inhibitors with improved potency and desirable selectivity profiles - known in this field as 'the tail approach' (there are 16 known human CA isozymes). ${ }^{1}$ Clinically used CA inhibitors include acetazolamide, methazolamide, ethoxazolamide, brinzolamide and dichlorophenamide while Indisulam is in Phase II clinical trials as an anticancer agent to treat solid tumours. ${ }^{6,7}$

\section{Insert Chemical Structures: group A}

For drug development the physiochemical properties (for example aqueous solubility, lipophilicity, stability, bioavailability) that make a compound a good drug must be considered alongside the goal to prepare compounds with desirable potency and selectivity profiles. ${ }^{9}$ For 
poorly soluble chemotherapeutic drugs that are administered by intravenous injection (such as the taxanes) this balance has been achieved indirectly through solubilizing vehicle formulations. Unfortunately the solubilizing additives themselves exhibit adverse effects outside those of the drug, leading to a requirement for co-treatment with other medications, long drug infusion times and sometimes even preventing the most relevant drug dose to be administered. ${ }^{10}$ The development of drug solubilizing strategies that are free from side effects caused by the formulation vehicle is thus of immense interest, particularly for chemotherapy applications - the recent development of $\mathrm{ABRAXANE}^{\circledR}$ (human albumin bound paclitaxel) is evidence for the success of this strategy. ${ }^{11}$

\section{Insert Chemical Structure Group B}

An alternative approach to equip drug molecules with improved solubility and tolerability is to attach carbohydrate moieties to generate glycoconjugates. ${ }^{12-15}$ This carbohydrate-based strategy has however not often received significant attention by the pharmaceutical industry, with sugars 'sidelined' owing to the stigma of both weak binding and a poor ability to cross cell membranes - leaving more traditional small organic molecules to be pursued for drug discovery. ${ }^{16}$ The cancer relevant hCA isozymes, unlike the physiologically dominant cytosolic hCA I and hCA II, are transmembrane proteins with extracellular enzyme active sites. Owing to the success of the 'tail approach' to generate potent CA inhibitors and the extracellular location of the hCA IX active site (circumventing the need for targeting drugs to cross cell membranes) we decided to explore tethering carbohydrate tails to the high-affinity $\mathrm{ArSO}_{2} \mathrm{NH}_{2}$ pharmacophore to synthesize sulfonamide glycoconjugates. We anticipated that the immense stereochemical diversity inherent to carbohydrate scaffolds might facilitate added opportunities to survey regions of biological space removed from the CA active-site 
core to exploit for improved CA affinity and isozyme selectivity. Despite the synthesis of a vast multitude of $\mathrm{CA}$ inhibitors utilising the tail strategy and the potential benefits of appending carbohydrate moieties to drug molecules, the combination of these two strategies to generate CA inhibitors is essentially unexplored - there is just a single literature example ${ }^{17}$ (nine compounds - which were not evaluated against cancer relevant CA isozymes). This relatively untouched medicinal chemistry landscape has served as the primary inspiration for the compounds synthesized and presented in the current study.

We have recently demonstrated the versatility of the 1,3-dipolar cycloaddition reaction $(1,3-$ DCR; 'click chemistry') to readily generate 1,4-disubstituted-1,2,3-triazole glycoconjugates from azido sugars and varied alkyne substrates (Scheme 1$).{ }^{18}$ This high degree of versatility has encouraged us to now explore 1,3-DCR as a novel 'click-tailing' strategy to append carbohydrate tails onto the $\mathrm{CA} \mathrm{ArSO}_{2} \mathrm{NH}_{2}$ recognition motif to generate glycoconjugate $\mathrm{CA}$ inhibitors. Specifically we report the synthesis of 28 benzene sulfonamides containing carbohydrate triazole tails. All compounds were investigated for their in vitro inhibition of hCA I, hCA II, and hCA IX. A number of derivatives were found to be selective for the cancer associated isozyme hCA IX.

\section{Insert Scheme 1}

\section{Results and Discussion}

\section{Chemistry}

To facilitate our synthetic strategy it was necessary to synthesize CA recognition scaffolds that possessed dual functionality, both (i) an anchor for reliable CA affinity (i.e. an aryl 
sulfonamide), and (ii) either a terminal alkyne or azide moiety to act as the complementary partner for the 1,3-DCR with our panel of sugar building blocks. Many ester and amide derivatives of 4-carboxy benzene sulfonamide (1) are potent (low nM) inhibitors of hCA II. The amide derivatives have also shown good in vivo activity and prolonged duration as antiglaucoma agents. ${ }^{19}$ The $N$-propynyl amide derivative $\mathbf{2}$ and $O$-propynyl ester derivative $\mathbf{3}$ were thus designed as the scaffolds for 1,3-DCR with a panel of azido sugars. Scaffold 2 was synthesized by HBTU mediated amide coupling of $\mathbf{1}$ with propargyl amine, while scaffold $\mathbf{3}$ was synthesized by carbodiimide mediated esterification of 1 with propargyl alcohol.

\section{Insert Chemical Structures Group C}

A panel of seven peracetylated azido sugars a-g were also prepared, the carbohydrate derived from: a glucose, b galactose, $\mathbf{c}$ arabinose, $\mathbf{d} \mathrm{N}$-acetyl glucosamine, e glucuronic acid and $\mathbf{f}$ methyl- $\alpha$-D-glucopyranoside, respectively (Figure 1). Glycosyl azides a-f were synthesized by the stereoselective bimolecular displacement of the halide substituent of glycosyl halides precursors with an azide nucleophile, ${ }^{20}$ while the methyl 2,3,4-tri-O-acetyl-6-azido-6-deoxy$\alpha$-D-glucopyranoside $\mathbf{g}$ was prepared from methyl- $\alpha$-D-glucopyranoside as previously described. ${ }^{21}$ The glycosyl halide precursors were prepared from the corresponding peracetates or purchased from commercial sources.

\section{Insert Figure 1}

Scaffolds $\mathbf{2}$ and $\mathbf{3}$ were each reacted with the azido sugar panel $\mathbf{a - g}$ to generate the amide and ester series of peracetylated glycoconjugates, $\mathbf{2 a - 2 g}$ and $\mathbf{3 a - 3 g}$, respectively (Scheme 2, Figure 2). When using catalyst loading as described by Sharpless, ${ }^{22}$ the triazole forming 
reaction was found to be sluggish, even at elevated temperatures. However, when using 10 $\mathrm{mol} \%$ of the $\mathrm{Cu}(\mathrm{I})$ source and $20 \mathrm{~mol} \%$ of ascorbate, triazole formation proceeded smoothly in all cases and under very mild conditions. Reactions were generally complete following 30 min of vigorous stirring (as evidenced by TLC). The $O$-acetate groups of the amide series 2a$2 \mathrm{~g}$ were subsequently removed using methanolic sodium methoxide to liberate the fully deprotected sugar analogues $\mathbf{2} \mathbf{a}^{\mathbf{\prime}} \mathbf{- 2} \mathbf{2} \mathbf{\prime}$ in quantitative or near quantitative yields. These basic reaction conditions proved unsuitable for the synthesis of the ester series $\mathbf{3} \mathbf{a}^{\mathbf{\prime}} \mathbf{- 3} \mathbf{g}^{\mathbf{9}}$, owing to the methoxide anion participating also in transesterification, whereby cleaving the $\mathrm{CA}$ anchor from the sugar triazole tail. More conveniently, the azido sugar panel were first de-Oacetylated to give the deprotected azido sugars $\mathbf{a}^{\prime}-\mathbf{g}$ ' $(\mathrm{R}=\mathrm{Ac}->\mathrm{R}=\mathrm{H})$ which were then reacted with scaffold $\mathbf{3}$ to give the deprotected sugar ester series $\mathbf{3} \mathbf{a}^{\mathbf{\prime}} \mathbf{-} \mathbf{3 g} \mathbf{g}$. All new compounds were characterized using $1 \mathrm{D}^{1} \mathrm{H}$ and ${ }^{13} \mathrm{C}$ NMR spectroscopy, 2D NMR spectroscopy (gCOSY, gHSQC, gHMBC as required), elemental analysis and HRMS.

\section{Insert Scheme 2}

\section{Insert Figure 2}

\section{Carbonic Anhydrase Inhibition}

hCA I, II, and IX enzyme inhibition data (determined by assaying the CA catalyzed hydration of $\left.\mathrm{CO}_{2}\right)^{23}$ for $\mathbf{2}, \mathbf{3}$ and the 28 new glycoconjugate sulfonamides are presented in Table 1. The selectivity ratios for inhibition of isozyme IX cf. I and II are also given in Table 1. Data for clinically used CA inhibitors acetazolamide (AZA), methazolamide (MZA), ethoxzolamide 
(EZA), dichlorophenamide (DCP), brinzolamide (BRZ) and Indisulam (IND) are included for comparison.

\section{Insert Table 1}

The parent amide scaffold $\mathbf{2}$ and ester scaffold $\mathbf{3}$ exhibited inhibition and isozyme selectivity similar to each other against the three hCA isozymes investigated. Compounds $\mathbf{2}$ and $\mathbf{3}$ had greatest efficacy at hCA II ( $K_{\mathrm{i}} \mathrm{s}$ of 47 and $45 \mathrm{nM}$, respectively), approximately 2 -fold weaker inhibition at hCA IX (113 and $104 \mathrm{nM}$, respectively) and an order of magnitude again weaker inhibition at hCA I (6100 and $4000 \mathrm{nM}$, respectively).

Isozyme hCA I. At hCA I the triazole linked carbohydrate moiety had minimal effect on inhibition, with 26 of the 28 compounds exhibiting affinity similarly ( $\mu \mathrm{M}$ range) to the parent compounds $\mathbf{2}$ and $\mathbf{3}$. The two notable exceptions were compounds $\mathbf{3} \mathbf{c}^{\mathbf{2}}$ and $\mathbf{3 e}$ ', both contained an ester linkage and a deprotected (free hydroxy) sugar. Compounds 3c' and 3e' were 400 to 500 -fold more potent than their parent ester scaffold $3\left(K_{\mathrm{i}} \mathrm{s}\right.$ of $7.7 \mathrm{nM}$ and 9.6 $\mathrm{nM}$, respectively) and more potent than any of the standard sulfonamide inhibitors tested. This result was extremely encouraging as it provided confirmation that the subtle structural differences in the sugar tail could indeed discriminate CA isozyme active site topology to substantially influence enzyme inhibition characteristics.

Isozyme hCA II. At hCA II the sugar triazole tail had a variable effect on CA inhibition - with some derivatives improved inhibition was observed while with others weaker inhibition was observed - when compared to the parent compounds $\mathbf{2}$ and $\mathbf{3}$. The ester linked deprotected sugars (3a'-3g') had tightly grouped $K_{\mathrm{i}} \mathrm{s}$, ranging from $5.8-8.6 \mathrm{nM}$, that were $\sim 5$ 
to 6-fold more potent than the parent 3. In striking contrast the amide linked deprotected sugars (2a'-2g') did not exhibit the same tight grouping of $K_{\mathrm{i}} \mathrm{s}$ and only the glucose and galactose derivatives (2a' and $\mathbf{2} \mathbf{b}^{\prime}$ ) paralleled the improved inhibition of their ester counterparts with $K_{\mathrm{i}} \mathrm{s}$ of 7.0 and $8.1 \mathrm{nM}$, respectively. The remaining amide linked deprotected sugars (compounds 20'-2g') had weaker inhibition than the parent $\mathbf{2}$, with $K_{\mathrm{i}}$ values ranging from $90-378 \mathrm{nM}$. For the ester linked series with the sugar acetates in place derivatives $\mathbf{3 b}, \mathbf{3 c}$ and $\mathbf{3 e}$ had $K_{\mathrm{i}} \mathrm{s}$ similar to the ester linked deprotected sugar derivatives, again $\sim 5$ to 6 -fold more potent than the parent $3\left(K_{\mathrm{i}} \mathrm{s}\right.$ ranging from $\left.6.8-7.3 \mathrm{nM}\right)$, while $\mathbf{3 g}$ had a similar $K_{\mathrm{i}}$ to the parent $\mathbf{3}(50 \mathrm{nM}$ vs. $45 \mathrm{nM})$ and 3a, 3d and $\mathbf{3 f}$ all exhibited weaker $K_{\mathrm{i}} \mathrm{s}$ than the parent 3 or deprotected sugar counterparts $\left(K_{\mathrm{i}} \mathrm{s}\right.$ ranging from $\left.119-423 \mathrm{nM}\right)$. For the acetylated sugars of the amide series (2a-2g) the derivative $\mathbf{2 g}$ had 2-fold higher inhibition than the deprotected sugar derivatives $\mathbf{2 g}$ ' and similar inhibition to parent amide $\mathbf{2}$. Derivatives 2d, 2e and 2f exhibited 6-, 50- and 36-fold higher inhibition, respectively, than their deprotected sugar counterparts, while derivatives $\mathbf{2 a}, \mathbf{2 b}$ and $\mathbf{2 c}$ were each weaker hCA II inhibitors than their deprotected sugar counterparts. In summary, for the seven different glycosyl triazole tails studied, the four groupings of amide, ester, sugar-OAc or sugar-OH, did not behave in a consistent direction with respect to hCA II inhibition. This in vitro data will be important for understanding future in vivo data, as it is clear that the acetates could serves as prodrugs in the in vivo environment. ${ }^{29}$ Similarly to the results for hCA I, these results again demonstrate that the sugar tails interrogate the enzyme active site with an intricacy of interactions that permit marked discrimination of enzyme inhibition.

Isozyme hCA IX. At hCA IX the parent compounds $\mathbf{2}$ and $\mathbf{3}$ had $K_{\mathrm{i}} \mathrm{s}$ of 113 and 104 $\mathrm{nM}$, respectively, approximately 2-fold weaker than hCA II inhibition and 40 to 50-fold more potent than hCA I inhibition. With five of the sugar tails $(\mathbf{b}, \mathbf{c}, \mathbf{e}, \mathbf{f}, \mathbf{g})$ there was at least one 
compound of the grouping (amide, ester, sugar-OAc or sugar-OH) that exhibited improved hCA IX inhibition over the parent scaffolds. The exceptions were the glucose tail a and the $\mathrm{N}$ acetyl glucosamine tail $\mathbf{d}$ in which the sugar triazole tail lead always to reduced inhibition when compared to the non-glycoconjugate parent scaffolds. For each sugar tail the amide sugar-OAc derivatives $(\mathbf{2 x})$ were more potent than sugar-OH derivatives $\left(\mathbf{2} \mathbf{x}^{\prime}\right)$ except for the glucuronic acid derivatives (2e' more potent than $\mathbf{2 e}$ ), while in the ester series this trend was completely reversed, with sugar-OH derivatives (3x') more potent than sugar-OAc derivatives $(\mathbf{3 x})$, again with the exception of the glucuronic acid derivatives (3e more potent than $\mathbf{3} \mathbf{e}^{\text {') }}$. Compounds $\mathbf{2 b}, \mathbf{3 b}, \mathbf{3} \mathbf{c}^{\prime}, \mathbf{2} \mathbf{e}^{\prime}, \mathbf{3 f}$ ', $\mathbf{3 g}$ and $\mathbf{3 g}$ ' were all stronger inhibitors of hCA IX than the parents 2 and 3, with $K_{\mathrm{i}}$ s ranging from $23-96 \mathrm{nM}$. The strongest hCA IX inhibitor was the amide linked deprotected glucuronic acid derivative $2 \mathrm{e}^{\prime}\left(K_{\mathrm{i}}=23 \mathrm{nM}\right)$. Importantly this compound was also 16.4-fold selective for hCA IX over hCA II and 100-fold selective over hCA I. Glucuronate $2 \mathbf{e}^{\prime}$ has a similar $K_{\mathrm{i}}$ to the sulfonamide indisulam $(24 \mathrm{nM})$ that is in phase II clinical trials as an anticancer agent, however indisulam is not selective for hCA IX whereas $\mathbf{2} \mathbf{e}^{\prime}$ is. There were several other compounds with mild hCA IX selectivity cf. hCA II, of note is the amide linked acetylated galactose derivative $\mathbf{2 b}$ with 6.2-fold selectivity (76 nM versus $470 \mathrm{nM}$ ). These results demonstrate that by tethering a sugar triazole tail onto the CA anchor pharmacophore it is possible to reverse the CA isozyme selectivity trends observed in the non-glycoconjugate parent compounds $\mathbf{2}$ and $\mathbf{3}$ and also all standard inhibitors - none of which are hCA IX selective.

\section{Conclusions}

Selective inhibition among isozymes is often a challenging hurdle in the drug discovery process, however to maximise the benefits of any future therapies involving CA inhibition, it is essential to develop isozyme-specific compounds. Conservation of active site structure and 
topology within the CA enzyme family has made it difficult to target subtle isozyme differences by rational drug design. Here we have explored 'click-tailing' carbohydrates onto the $\mathrm{ArSO}_{2} \mathrm{NH}_{2} \mathrm{CA}$ pharmacophore as a drug solubilizing and isozyme differentiating strategy. This work presents a new class of CA inhibitors comprising triazole-tethered carbohydrate tails, the first of which to have been generated through 'click chemistry'. The qualitative structure-activity demonstrated that the stereochemical diversity within the carbohydrate tails effectively interrogated the CA active site topology, generating in some instances inhibitors with hCA IX selectivity - an important outcome in the quest for potential cancer therapy applications. Sugar tails may therefore prove a valuable approach to generate CA isozyme selective compounds. A powerful example is glucose (2a, 2a', 3a, 3a') and galactose (2b, 2b', 3b, 3b') epimer derivatives that have drastically different inhibition and selectivity profiles yet the sole stereochemical difference is over 14 atoms removed from the sulfonamide moiety. The relative ease of synthetic access to azido sugars coupled with the venerability of the cycloaddition reaction has provided an efficient route to the carbohydratebased CA inhibitors. We intend to more fully explore this "click tailing' strategy for the development of CA inhibitors for therapeutic investigation. 


\section{Experimental section}

Chemistry. Reagents were purchased from Sigma Aldrich and Fluka chemical companies and were used without further purification. Solvents were dried and distilled where necessary prior to use, or purchased as such from Sigma Aldrich. Reactions were monitored by TLC using Merck $\mathrm{F}_{60} 0_{254}$ silica plates with visualization of product bands by UV fluorescence $(\lambda=254 \mathrm{~nm})$ and charring by $10 \% \mathrm{v} / \mathrm{v}$ ethanolic $\mathrm{H}_{2} \mathrm{SO}_{4}$. Flash chromatography was performed on Merck flash silica gel $(0.04 \mathrm{~mm}-0.06 \mathrm{~mm})$. Melting points were acquired on a Gallenkamp melting point apparatus and are reported as uncorrected. NMR $\left({ }^{1} \mathrm{H}\right.$ and ${ }^{13} \mathrm{C}$ $\left.\left\{{ }^{1} \mathrm{H}\right\}\right)$ spectra were recorded on a Varian Unity $400 \mathrm{MHz}$ spectrometer at room temperature using DMSO- $d_{6}$ solvent unless otherwise stated. Chemical shifts are reported in $\delta(\mathrm{ppm})$ from a TMS internal standard $(0.0 \mathrm{ppm})$. Coupling constants $(J)$ are reported in hertz. High resolution electrospray ionisation mass spectra were performed in negative ion mode on an Apex III Bruker Daltonics 4.7T Fourier Transform mass spectrometer (FTMS) fitted with an Apollo ESI source.

$\boldsymbol{N}$-(Prop-2-ynyl)-4-sulfamoylbenzamide (2). To a stirring solution of 1 (2.0 g, 9.9 mmol) and propargyl amine (0.64 mL, $9.9 \mathrm{mmol}, 1.0$ equiv) in dry DMF (40 mL) was successively added $\quad N$-hydroxybenzotriazole monohydrate $(0.94$ g, $6.6 \mathrm{mmol}, \quad 0.6$ equiv), diisopropylethylamine (1.7 mL, 9.9 mmol, 1.0 equiv) and HBTU (3.8 g, 9.9 mmol, 1.0 equiv). The deep yellow solution was stirred at room temperature under nitrogen for 1 hour when found complete by TLC. The mixture was concentrated under reduced pressure and ethyl acetate $(40 \mathrm{~mL})$ was added. The organic extract was washed with water $(40 \mathrm{~mL})$ and back extracted with ethyl acetate $(3 \times 40 \mathrm{~mL})$. The organic extracts were combined, and washed with brine $(50 \mathrm{~mL})$. The organic layer was dried $\left(\mathrm{MgSO}_{4}\right)$, filtered and evaporated to a crude white solid. Recrystallization from hot methanol:water (9:1) afforded the title compound 2 as 
white crystalline solid (1.9 g, $8.2 \mathrm{mmol}, 82 \%) . R_{f} 0.58$ (100\% EtOAc); mp $216-217{ }^{\circ} \mathrm{C}$ (decomp). ${ }^{1} \mathrm{H}$ NMR (400 MHz, DMSO-d $): \delta 3.12\left(\mathrm{t},{ }^{4} J_{\mathrm{CH}-\mathrm{CH}}=2.4 \mathrm{~Hz}, 1 \mathrm{H}\right.$, propynyl $\left.\mathrm{CH}\right)$, $4.05\left(\mathrm{dd},{ }^{2} J_{\mathrm{CH}-\mathrm{NH}}=5.6 \mathrm{~Hz},{ }^{4} J_{\mathrm{CH}-\mathrm{CH}}=2.8 \mathrm{~Hz}, 2 \mathrm{H}\right.$, propynyl $\left.\mathrm{CH}_{2}\right), 7.45\left(\right.$ br s, $\left.2 \mathrm{H}, \mathrm{SO}_{2} \mathrm{NH}_{2}\right)$, $7.86-7.98(\mathrm{~m}, 4 \mathrm{H}, \mathrm{Ph}), 9.09\left(\mathrm{t},{ }^{3} \mathrm{~J}_{\mathrm{NH}-\mathrm{CH}}=5.6 \mathrm{~Hz}, 1 \mathrm{H}, \mathrm{CONH}\right) ;{ }^{13} \mathrm{C}\left\{{ }^{1} \mathrm{H}\right\} \mathrm{NMR}(100 \mathrm{MHz}$, DMSO- $d_{6}$ ); $\delta 29.03$ (propynyl $\mathrm{CH}_{2}$ ), 73.81 (propynyl $\mathrm{CH}$ ), 81.67 (propynyl $\mathrm{C}$ ), $126.37(\mathrm{Ph}$ $\mathrm{CH}), 128.64(\mathrm{Ph} \mathrm{CH}), 137.34(\mathrm{Ph} \mathrm{C}), 147.14(\mathrm{Ph} \mathrm{C}), 165.62(\mathrm{C}=\mathrm{O})$. HRMS (ESI) calcd for $\mathrm{C}_{10} \mathrm{H}_{9} \mathrm{~N}_{2} \mathrm{O}_{3} \mathrm{~S}^{-}:$237.033936; found: 237.034388. Anal. $\left(\mathrm{C}_{10} \mathrm{H}_{10} \mathrm{~N}_{2} \mathrm{O}_{3} \mathrm{~S}\right) \mathrm{C}, \mathrm{H}, \mathrm{N}, \mathrm{S}$.

Prop-2-ynyl 4-sulfamoylbenzoate (3). To a stirring solution of 1 (2.0 g, $9.9 \mathrm{mmol})$ in dry DMF (40 mL) was successively added propargyl alcohol (1.17 mL, 19.8 mmol, 2.0 equiv), $\mathrm{Et}_{3} \mathrm{~N}$ (2.8 mL, $19.9 \mathrm{mmol}, 2.0$ equiv) and $\mathrm{EDC}$ (1.9 g, $9.9 \mathrm{mmol}, 1.0$ equiv). The solution was stirred at room temperature under $\mathrm{N}_{2}$ for an additional 4 hours. The mixture was then concentrated under reduced pressure and ethyl acetate $(40 \mathrm{~mL})$ was added. The organic extract was washed with saturated aqueous $\mathrm{NaHCO}_{3}(40 \mathrm{~mL})$ and back extracted with ethyl acetate $(40 \mathrm{~mL})$. The organic layers were combined and washed with brine $(40 \mathrm{~mL})$, dried $\left(\mathrm{MgSO}_{4}\right)$, filtered and evaporated. The crude oil was purified by flash silica chromatography (1:1 EtOAc:hexanes) to afford the title compound $\mathbf{3}$ as white crystalline solid $(0.91 \mathrm{~g}, 3.8$ mmol, $38 \%$ \%). $R_{f} 0.38$ (6:4 EtOAc:hexanes); mp 110 - $111{ }^{\circ} \mathrm{C} .{ }^{1} \mathrm{H}$ NMR (400 MHz, DMSO$\left.d_{6}\right): \delta 3.63\left(\mathrm{t},{ }^{4} J_{\mathrm{CH}-\mathrm{CH}}=2.4 \mathrm{~Hz}, 1 \mathrm{H}\right.$, propynyl $\left.\mathrm{CH}\right), 4.97\left(\mathrm{~d},{ }^{4} J_{\mathrm{CH}-\mathrm{CH}}=2.8 \mathrm{~Hz}, 2 \mathrm{H}\right.$, propynyl $\mathrm{CH}_{2}$ ), 7.55 (br s, 2H, $\mathrm{SO}_{2} \mathrm{NH}_{2}$ ), $7.93-8.13$ (m, 4H, Ph); ${ }^{13} \mathrm{C}\left\{{ }^{1} \mathrm{H}\right\}$ NMR (100 MHz, DMSO$d_{6}$ ): $\delta 53.00$ (propynyl $\mathrm{CH}_{2}$ ), 78.12 (propynyl $\mathrm{CH}$ ), 78.28 (propynyl C), $126.16(\mathrm{Ph} \mathrm{CH})$, $130.03(\mathrm{Ph} \mathrm{CH}), 131.68$ (Ph C), 148.32 (Ph C), $164.05(\mathrm{C}=\mathrm{O})$. HRMS (ESI) calcd for $\mathrm{C}_{10} \mathrm{H}_{8} \mathrm{NO}_{4} \mathrm{~S}^{-}: 238.017952$. Found: 238.017407. Anal. $\left(\mathrm{C}_{10} \mathrm{H}_{9} \mathrm{NO}_{4} \mathrm{~S}\right) \mathrm{C}, \mathrm{H}, \mathrm{N}, \mathrm{S}$.

Synthesis of glycosyl triazole aryl sulfonamides (2a -g, 3a' - g'): General Procedure 1. A mixture of the azide (1.0 equiv) and acetylene (1.0 equiv) was suspended in a tert-butyl 
alcohol and water $(1: 1,0.2-0.5 \mathrm{M}$ final concentration). A solution of sodium ascorbate $(0.2$ equiv) in water, followed by a solution $\mathrm{CuSO}_{4} .5 \mathrm{H}_{2} \mathrm{O}$ (0.1 equiv) in water was successively added. The bright yellow suspension was stirred vigorously at $40{ }^{\circ} \mathrm{C}$ until TLC indicated reaction completion (generally within 2 hours). The mixture was evaporated under reduced pressure and the resulting residue was purified by flash chromatography to yield pure material.

Preparation of deprotected glycosyl triazole aryl sulfonamides (2a' - g' and 3a'-g'): General Procedure 2. Compounds 2a' - g' were prepared by the treating the acetylated precursors $2 \mathbf{a}-\mathbf{g}$ (final concentration of $\sim 0.1-0.2 \mathrm{M}$ ) with dry methanolic sodium methoxide (final pH 9 - 12). Reactions were found to be complete within 30 minutes by TLC. Neutralization of the solution by Amberlite IR-120 ion exchange resin, followed by filtration and evaporation of the filtrate to dryness afforded pure material by NMR. Likewise, the analogous esters $\mathbf{3 a}$ ' $-\mathbf{g}$ ' were prepared by deprotecting the peracetylated glycosyl azides $\mathbf{a}-$ f or the 6-deoxy-6-azido glucoside $\mathbf{g}$ in the same way as described above, prior to the cycloaddition reaction with $\mathbf{3}$ (see scheme 2).

\section{4-(\{[4-(Aminosulfonyl)benzoyl]amino\}methyl-1-(2',3',4',6'-tetra-O-acetyl- $\beta$-D-}

glucopyranosyl)-1-H-1,2,3-triazole (2a). The title compound was prepared according to the General Procedure 1 and isolated as white solid (172 mg, $0.28 \mathrm{mmol}, 86 \%) . R_{f} 0.15(100 \%$ EtOAc); mp $198-199{ }^{\circ} \mathrm{C} .{ }^{1} \mathrm{H}$ NMR (400 MHz, DMSO-d 6 ): $\delta 1.75$ (s, 3H, OAc CH ), 1.92 (s, 3H, OAc $\mathrm{CH}_{3}$ ), 1.95 (s, 3H OAc $\mathrm{CH}_{3}$ ), 1.99 (s, 3H OAc $\left.\mathrm{CH}_{3}\right), 4.01-4.10$ (m, 2H, $\mathrm{H}_{6}$, $\mathrm{H}_{6}$ ") $4.27-4.33\left(\mathrm{~m}, 1 \mathrm{H}, \mathrm{H}_{5}\right), 4.50\left(\mathrm{~d},{ }^{3} J_{\mathrm{CH}-\mathrm{NH}}=6.0 \mathrm{~Hz}, 2 \mathrm{H}, \mathrm{CH}_{2} \mathrm{NH}\right), 5.11-5.16\left(\mathrm{~m}, 1 \mathrm{H}, \mathrm{H}_{4}{ }^{\prime}\right)$, $5.47-5.52\left(\mathrm{~m}, 1 \mathrm{H}, \mathrm{H}_{3}{ }^{\prime}\right), 5.61-5.66\left(\mathrm{~m}, 1 \mathrm{H}, \mathrm{H}_{2}{ }^{\prime}\right), 6.28\left(\mathrm{~d},{ }^{3} J_{1^{\prime}-2^{\prime}}=9.2 \mathrm{~Hz}, 1 \mathrm{H}, \mathrm{H}_{1^{\prime}}\right), 7.44(\mathrm{~s}$, $\left.2 \mathrm{H}, \mathrm{SO}_{2} \mathrm{NH}_{2}\right), 7.86-7.99(\mathrm{~m}, 4 \mathrm{H}, \mathrm{Ph} \mathrm{H}), 8.26(\mathrm{~s}, 1 \mathrm{H}$, triazole $\mathrm{H}), 9.22\left(\mathrm{t},{ }^{3} J_{\mathrm{NH}-\mathrm{CH}}=5.2 \mathrm{~Hz}\right.$, 
$\left.1 \mathrm{H}, \mathrm{CH}_{2} \mathrm{NH}\right) ;{ }^{13} \mathrm{C}\left\{{ }^{1} \mathrm{H}\right\}$ NMR $\left(100 \mathrm{MHz}, \mathrm{DMSO}-d_{6}\right): \delta 20.55\left(\mathrm{OAc} \mathrm{CH}_{3}\right), 20.87\left(\mathrm{OAc} \mathrm{CH}_{3}\right)$, $21.00\left(\mathrm{OAc} \mathrm{CH}_{3}\right), 21.14\left(\mathrm{OAc}_{\mathrm{CH}}\right), 35.46\left(\mathrm{CH}_{2} \mathrm{NH}\right), 62.38\left(\mathrm{C}_{6^{\prime}}\right), 68.13\left(\mathrm{C}_{4}{ }^{\prime}\right), 70.69\left(\mathrm{C}_{3^{\prime}}\right)$, $72.91\left(\mathrm{C}_{2}\right), 73.95\left(\mathrm{C}_{5}\right), 84.46\left(\mathrm{C}_{1}\right), 122.82$ (triazole $\left.\mathrm{CH}\right), 126.31(\mathrm{Ph} \mathrm{CH}), 128.64(\mathrm{Ph} \mathrm{CH})$, $137.63(\mathrm{Ph} \mathrm{C}), 146.24$ (triazole $\mathrm{C}$ or $\mathrm{Ph} \mathrm{C}), 146.96$ (triazole $\mathrm{C}$ or $\mathrm{Ph} \mathrm{C}), 166.05(\mathrm{C}=\mathrm{O}), 169.23$ $(\mathrm{C}=\mathrm{O}), 170.11(\mathrm{C}=\mathrm{O}), 170.34(\mathrm{C}=\mathrm{O}), 170.83(\mathrm{C}=\mathrm{O})$. HRMS (ESI) calcd for $\mathrm{C}_{24} \mathrm{H}_{28} \mathrm{~N}_{5} \mathrm{O}_{12} \mathrm{~S}^{-}$: 610.146066. Found: 610.147077. Anal. $\left(\mathrm{C}_{24} \mathrm{H}_{29} \mathrm{~N}_{5} \mathrm{O}_{12} \mathrm{~S} \bullet 0.5 \mathrm{H}_{2} \mathrm{O}\right) \mathrm{C}, \mathrm{H}, \mathrm{N}$.

\section{4-(\{[4-(Aminosulfonyl)benzoyl]amino\} methyl-1-( $\beta$-D-glucopyranosyl)-1-H-1,2,3-triazole}

(2a'). Title compound was prepared according to General Procedure 2 and isolated as white solid (71 mg, $0.16 \mathrm{mmol}, \sim 100 \%)$; mp $205-210{ }^{\circ} \mathrm{C}$ (decomp). $R_{f} 0.15\left(1: 9 \mathrm{H}_{2} \mathrm{O}: \mathrm{CH}_{3} \mathrm{CN}\right) .{ }^{1} \mathrm{H}$ NMR (400 MHz, $\left.\mathrm{D}_{2} \mathrm{O}\right): \delta 3.46-3.51\left(\mathrm{~m}, 1 \mathrm{H}, \mathrm{H}_{4}{ }^{\prime}\right), 3.55-3.60\left(\mathrm{~m}, 1 \mathrm{H}, \mathrm{H}_{3}{ }^{\prime}\right), 3.58-3.66(\mathrm{~m}$, $\left.2 \mathrm{H}, \mathrm{H}_{5}, \mathrm{H}_{6}{ }^{\prime}\right), 3.74-3.79$ (m, $\left.1 \mathrm{H}, \mathrm{H}_{6}{ }^{\prime}\right), 3.84-3.88$ (m, $\left.1 \mathrm{H}, \mathrm{H}_{2}\right), 4.59$ (s, 2H, $\left.\mathrm{CH}_{2} \mathrm{NH}\right), 5.61$ $\left(\mathrm{d},{ }^{3} J_{1^{\prime}-2^{\prime}}=8.8 \mathrm{~Hz}, 1 \mathrm{H}, \mathrm{H}_{1^{\prime}}\right), 7.80-7.88(\mathrm{~m}, 4 \mathrm{H}, \mathrm{Ph}), 8.07(\mathrm{~s}, 1 \mathrm{H}$, triazole $\mathrm{CH}) ;{ }^{13} \mathrm{C}\left\{{ }^{1} \mathrm{H}\right\}$ NMR (100 MHz, 2\% D $\mathrm{D}_{2} \mathrm{O}$ in DMSO-d $\left.d_{6}\right): \delta 35.19\left(\mathrm{CH}_{2} \mathrm{NH}\right), 60.53\left(\mathrm{C}_{6}\right), 69.07\left(\mathrm{C}_{4}{ }^{\prime}\right), 72.41$ $\left(\mathrm{C}_{2}{ }^{\prime}\right), 76.02\left(\mathrm{C}_{3^{\prime}}\right), 79.04\left(\mathrm{C}_{5},\right), 87.59\left(\mathrm{C}_{1}{ }^{\prime}\right), 123.36$ (triazole $\left.\mathrm{CH}\right), 126.44(\mathrm{Ph} \mathrm{CH}), 128.44(\mathrm{Ph}$ $\mathrm{CH}), 137.71$ (Ph C), 144.35 (triazole $\mathrm{C}$ or $\mathrm{Ph} \mathrm{C}), 144.94$ (triazole $\mathrm{C}$ or $\mathrm{Ph} \mathrm{C}), 169.54(\mathrm{C}=\mathrm{O})$. HRMS (ESI) calcd for $\mathrm{C}_{16} \mathrm{H}_{20} \mathrm{~N}_{5} \mathrm{O}_{8} \mathrm{~S}^{-}:$442.10387. Found: 442.103042.

\section{4-(\{[4-(Aminosulfonyl)benzoyl]amino\}methyl-1-(2',3',4',6'-tetra-O-acetyl- $\beta$-D-}

galactopyranosyl)-1-H-1,2,3-triazole (2b). The title compound was prepared according to the General Procedure 1 and isolated as white solid (174 mg, $0.28 \mathrm{mmol}, 87 \%) . R_{f} 0.11(8: 2$ EtOAc:hexanes); mp $161-162{ }^{\circ} \mathrm{C}$ (decomp). ${ }^{1} \mathrm{H}$ NMR (400 MHz, DMSO-d $\left.d_{6}\right): \delta 1.78$ (s, 3H, $\mathrm{OAc} \mathrm{CH}_{3}$ ), 1.91 (s, 3H, OAc $\mathrm{CH}_{3}$ ), 1.95 (s, 3H, OAc $\mathrm{CH}_{3}$ ), 2.15 (s, 3H, OAc $\mathrm{CH}_{3}$ ), 3.98 (dd, $\left.{ }^{2} J_{6^{\prime}-6^{\prime}}=11.6 \mathrm{~Hz},{ }^{3} J_{6^{\prime}-5},=7.6 \mathrm{~Hz}, 1 \mathrm{H}, \mathrm{H}_{6^{\prime}}\right), 4.10\left(\mathrm{dd},{ }^{2} J_{6^{\prime}}{ }^{\prime}-6^{\prime}=11.6 \mathrm{~Hz},{ }^{3} J_{6^{\prime}}{ }^{-5},=5.2 \mathrm{~Hz}, 1 \mathrm{H}\right.$, $\left.\mathrm{H}_{6}{ }^{\prime}\right), 4.51\left(\mathrm{~d},{ }^{3} J_{\mathrm{CH}-\mathrm{NH}}=5.5 \mathrm{~Hz}, 2 \mathrm{H}, \mathrm{CH}_{2} \mathrm{NH}\right), 4.52-4.55\left(\mathrm{~m}, 1 \mathrm{H}, \mathrm{H}_{5}{ }^{\prime}\right), 5.38-5.43(\mathrm{~m}, 2 \mathrm{H}$, 
$\left.\mathrm{H}_{3^{\prime}}, \mathrm{H}_{4}{ }^{\prime}\right), 5.55-5.60\left(\mathrm{~m}, 2 \mathrm{H}, \mathrm{H}_{2}{ }^{\prime}\right), 6.21\left(\mathrm{~d},{ }^{3} \mathrm{~J}_{1^{\prime}-2^{\prime}}=9.2 \mathrm{~Hz}, 1 \mathrm{H}, \mathrm{H}_{1^{\prime}}\right), 7.45\left(\mathrm{~s}, 2 \mathrm{H}, \mathrm{SO}_{2} \mathrm{NH}_{2}\right)$, $7.86-7.99(\mathrm{~m}, 4 \mathrm{H}, \mathrm{Ph}), 8.16(\mathrm{~s}, 1 \mathrm{H}$, triazole $\mathrm{H}), 9.19\left(\mathrm{t},{ }^{3} J_{\mathrm{NH}-\mathrm{CH}}=5.1 \mathrm{~Hz}, 1 \mathrm{H}, \mathrm{CH}_{2} \mathrm{NH}\right) ;{ }^{13} \mathrm{C}$ $\left\{{ }^{1} \mathrm{H}\right\}$ NMR $\left(100 \mathrm{MHz}, \mathrm{DMSO}-d_{6}\right): \delta 20.69\left(\mathrm{OAc} \mathrm{CH}_{3}\right), 20.98\left(\mathrm{OAc} \mathrm{CH}_{3}\right), 21.10\left(\mathrm{OAc} \mathrm{CH}_{3}\right)$, $21.17\left(\mathrm{OAc} \mathrm{CH}_{3}\right), 35.46\left(\mathrm{CH}_{2} \mathrm{NH}\right), 62.25\left(\mathrm{C}_{6}{ }^{\prime}\right), 68.01\left(\mathrm{C}_{4}{ }^{\prime}\right), 68.30\left(\mathrm{C}_{3}\right), 71.14\left(\mathrm{C}_{2}\right), 73.63$ $\left(\mathrm{C}_{5},\right), 84.85\left(\mathrm{C}_{1}{ }^{\prime}\right), 123.13($ triazole $\mathrm{CH}), 126.29(\mathrm{Ph} \mathrm{CH}), 128.66(\mathrm{Ph} \mathrm{CH}), 137.63(\mathrm{Ph} \mathrm{C})$, 145.97 (triazole $\mathrm{C}$ or $\mathrm{Ph} \mathrm{C}), 147.05$ (triazole $\mathrm{C}$ or $\mathrm{Ph} \mathrm{C}), 165.89(\mathrm{C}=\mathrm{O}), 169.18(\mathrm{C}=\mathrm{O}), 170.12$ $(\mathrm{C}=\mathrm{O}), 170.59 \mathrm{C}=\mathrm{O}), 170.66(\mathrm{C}=\mathrm{O})$. HRMS (ESI) calcd for $\mathrm{C}_{24} \mathrm{H}_{28} \mathrm{~N}_{5} \mathrm{O}_{12} \mathrm{~S}^{-}: 610.146066$. Found: 610.147458. Anal. $\left(\mathrm{C}_{24} \mathrm{H}_{29} \mathrm{~N}_{5} \mathrm{O}_{12} \mathrm{~S} \bullet 0.5 \mathrm{H}_{2} \mathrm{O}\right) \mathrm{C}, \mathrm{H}, \mathrm{N}$.

4-(4-Sulfamoylbenzamido)methyl-1-( $\beta$-D-galactopyranosyl)-1-H-1,2,3-triazole (2b'). The title compound was prepared according to General Procedure 2 and isolated as white foam (72 $\mathrm{mg}, 0.16 \mathrm{mmol}, \sim 100 \%) . R_{f} 0.13\left(1: 9 \mathrm{H}_{2} \mathrm{O}: \mathrm{CH}_{3} \mathrm{CN}\right) .{ }^{1} \mathrm{H} \mathrm{NMR}\left(400 \mathrm{MHz}, \mathrm{D}_{2} \mathrm{O}\right): \delta 3.64-$ $3.65\left(\mathrm{~m}, 2 \mathrm{H}, \mathrm{H}_{6^{\prime}}, \mathrm{H}_{6^{\prime}}\right), 3.74\left(\mathrm{dd},{ }^{3} J_{3^{\prime}-{ }^{\prime}},=9.6 \mathrm{~Hz},{ }^{3} J_{3^{\prime}-4},=3.2 \mathrm{~Hz}, 1 \mathrm{H}, \mathrm{H}_{3^{\prime}}\right), 3.87\left(\mathrm{~m}, 1 \mathrm{H}, \mathrm{H}_{5^{\prime}}\right)$, $3.95-3.96\left(\mathrm{dd},{ }^{3} J_{4^{\prime}-3^{\prime}},=3.4 \mathrm{~Hz},{ }^{3} J_{4^{\prime}-5},=0.8 \mathrm{~Hz}, 1 \mathrm{H}, \mathrm{H}_{4}{ }^{\prime}\right), 4.05-4.10\left(\mathrm{~m}, 1 \mathrm{H}, \mathrm{H}_{2}{ }^{\prime}\right), 4.59(\mathrm{~s}$, $\left.2 \mathrm{H}, \mathrm{CH}_{2} \mathrm{NH}\right), 5.55\left(\mathrm{~d}, 1 \mathrm{H},{ }^{3} J_{1^{\prime}-2}{ }^{\prime}=8.8 \mathrm{~Hz}, 1 \mathrm{H}, \mathrm{H}_{1}{ }^{\prime}\right), 7.80-7.88(\mathrm{~m}, 4 \mathrm{H}, \mathrm{Ph}), 8.11(\mathrm{~s}, 1 \mathrm{H}$, triazole $\mathrm{CH}) ;{ }^{13} \mathrm{C}\left\{{ }^{1} \mathrm{H}\right\}$ NMR $\left(100 \mathrm{MHz}, \mathrm{D}_{2} \mathrm{O}\right): \delta 35.24\left(\mathrm{CH}_{2} \mathrm{NH}\right), 60.99\left(\mathrm{C}_{6}{ }^{\prime}\right), 68.71\left(\mathrm{C}_{4}{ }^{\prime}\right)$, $69.91\left(\mathrm{C}_{2}{ }^{\prime}\right), 73.06\left(\mathrm{C}_{3^{\prime}}\right), 78.45\left(\mathrm{C}_{5}\right), 88.20\left(\mathrm{C}_{1}{ }^{\prime}\right), 123.05($ triazole $\mathrm{CH}), 126.44(\mathrm{Ph} \mathrm{H}), 128.44$ ( $\mathrm{Ph} \mathrm{H}), 137.72(\mathrm{Ph} \mathrm{C}), 144.32$ ( $\mathrm{Ph} \mathrm{C}$ or triazole $\mathrm{C}$ ), 145.63 (triazole $\mathrm{C}$ or $\mathrm{Ph} \mathrm{C}$ ), 169.53 $(\mathrm{C}=\mathrm{O})$. HRMS (ESI) calcd for $\mathrm{C}_{16} \mathrm{H}_{20} \mathrm{~N}_{5} \mathrm{O}_{8} \mathrm{~S}^{-}: 442.103807$. Found: 442.103051 .

\section{4-(\{[4-(Aminosulfonyl)benzoyl]amino\} methyl-1-(2',3',4'-tri-O-acetyl- $\beta$-D-}

arabinopyranosyl)-1-H-1,2,3-triazole (2c). The title compound was prepared according to the General Procedure 1 and isolated as off-white solid (147 mg, $0.27 \mathrm{mmol}, 82 \%) . R_{f} 0.10$ (8:2 EtOAc:hexanes); mp 125 - $126{ }^{\circ} \mathrm{C} .{ }^{1} \mathrm{H}$ NMR (400 MHz, DMSO-d $\left.)_{6}\right): \delta 1.78$ (s, 3H, OAc $\left.\mathrm{CH}_{3}\right), 1.92(\mathrm{~s}, 3 \mathrm{H}, \mathrm{OAc} \mathrm{CH}), 2.12\left(\mathrm{~s}, 3 \mathrm{H}, \mathrm{OAc} \mathrm{CH}_{3}\right), 3.99\left(\mathrm{dd},{ }^{2} J_{5}{ }^{\prime}-5,=13.2 \mathrm{~Hz},{ }^{3} J_{5}{ }^{\prime}-4,=2.0\right.$ 
$\left.\mathrm{Hz}, 1 \mathrm{H}, \mathrm{H}_{5}\right), 4.13\left(\mathrm{dd}, 1 \mathrm{H},{ }^{2} J_{5^{\prime}-5},=13.4 \mathrm{~Hz},{ }^{3} J_{5^{\prime},-4^{\prime}}=1.3 \mathrm{~Hz}, 1 \mathrm{H}, \mathrm{H}_{5^{\prime}}\right), 4.51\left(\mathrm{~d},{ }^{3} J_{\mathrm{CH}-\mathrm{NH}}=5.6\right.$ $\left.\mathrm{Hz}, 1 \mathrm{H}, \mathrm{CH}_{2} \mathrm{NH}\right), 5.27-5.28\left(\mathrm{~m}, 1 \mathrm{H}, \mathrm{H}_{4^{\prime}}\right), 5.36\left(\mathrm{dd}^{3}{ }^{3} \mathrm{~J}_{3^{\prime}-2^{\prime}}=10.4 \mathrm{~Hz},{ }^{3} J_{3^{\prime}-4^{\prime}}=5.2 \mathrm{~Hz}, 1 \mathrm{H}\right.$, $\left.\mathrm{H}_{3^{\prime}}\right), 5.55-5.59\left(\mathrm{~m}, 1 \mathrm{H}, \mathrm{H}_{2^{\prime}}\right), 6.07\left(\mathrm{~d},{ }^{3} J_{1^{\prime}-2^{\prime}}=9.2 \mathrm{~Hz}, 1 \mathrm{H}, \mathrm{H}_{1^{\prime}}\right), 7.45\left(\mathrm{br} \mathrm{s}, 2 \mathrm{H}, \mathrm{SO}_{2} \mathrm{NH}_{2}\right)$, $7.86-7.99(\mathrm{~m}, 4 \mathrm{H}, \mathrm{Ph} \mathrm{H}), 8.21(\mathrm{~s}, 1 \mathrm{H}$, triazole $\mathrm{H}), 9.18\left(\mathrm{t},{ }^{3} J_{\mathrm{NH}-\mathrm{CH}}=5.2 \mathrm{~Hz}, 1 \mathrm{H}, \mathrm{CH}_{2} \mathrm{NH}\right)$; ${ }^{13} \mathrm{C}\left\{{ }^{1} \mathrm{H}\right\}$ NMR (100 MHz, DMSO- $\left.d_{6}\right): \delta 20.70\left(\mathrm{OAc} \mathrm{CH}_{3}\right), 21.06\left(\mathrm{OAc} \mathrm{CH}_{3}\right), 21.40(\mathrm{OAc}$ $\left.\mathrm{CH}_{3}\right), 67.12\left(\mathrm{C}_{5^{\prime}}\right), 68.57\left(\mathrm{C}_{4}\right), 68.60\left(\mathrm{C}_{3^{\prime}}\right), 71.05\left(\mathrm{C}_{2}\right), 85.40\left(\mathrm{C}_{1}\right)$ ), 123.04 (triazole $\left.\mathrm{CH}\right)$, 126.29 (Ph CH), $128.65(\mathrm{Ph} \mathrm{CH}), 137.65(\mathrm{Ph} \mathrm{C}), 145.92$ (triazole $\mathrm{C}$ or $\mathrm{Ph} \mathrm{C}$ ), 147.04 (triazole $\mathrm{C}$ or Ph $\mathrm{C}), 165.89(\mathrm{C}=\mathrm{O}), 169.22(\mathrm{C}=\mathrm{O}) 170.20(\mathrm{C}=\mathrm{O}) 170.55(\mathrm{C}=\mathrm{O})$. HRMS (ESI) calcd for $\mathrm{C}_{21} \mathrm{H}_{24} \mathrm{~N}_{5} \mathrm{O}_{10} \mathrm{~S}:$ :538.124936. Found: 538.125762. Anal. $\left(\mathrm{C}_{21} \mathrm{H}_{26} \mathrm{~N}_{5} \mathrm{O}_{10} \mathrm{~S} \bullet 0.5 \mathrm{H}_{2} \mathrm{O}\right) \mathrm{C}, \mathrm{N} ; \mathrm{H}$ : calcd, 4.78 ; found, 4.17

\section{4-(\{[4-(Aminosulfonyl)benzoyl]amino\} methyl-1-(ß-D-garabinopyranosyl)-1-H-1,2,3-}

triazole (2'). The title compound was prepared according to General Procedure 2 and isolated as pale yellow solid (77 mg, $0.19 \mathrm{mmol}, \sim 100 \%) . R_{f} 0.26$ (1:9 EtOAc:MeOH); mp $210-212{ }^{\circ} \mathrm{C}$ (decomp). ${ }^{1} \mathrm{H}$ NMR $\left(400 \mathrm{MHz}, 1: 9\right.$ DMSO-d $\left.d_{6}: \mathrm{D}_{2} \mathrm{O}\right): \delta 3.74\left(\mathrm{dd},{ }^{3} J_{3^{\prime}-2},=10 \mathrm{~Hz}\right.$, $\left.{ }^{3} J_{3^{\prime}-4^{\prime}}=3.6 \mathrm{~Hz}, 1 \mathrm{H}, \mathrm{H}_{3^{\prime}}\right), 3.80-3.83\left(\mathrm{~m}, 1 \mathrm{H}, \mathrm{H}_{5^{\prime}}\right), 3.93-3.96\left(\mathrm{~m}, 2 \mathrm{H}, \mathrm{H}_{4}{ }^{\prime}, \mathrm{H}_{5^{\prime}}\right), 4.06-4.11$ (m, $\left.1 \mathrm{H}, \mathrm{H}_{2}{ }^{\prime}\right), 4.59\left(\mathrm{~s}, 2 \mathrm{H}, \mathrm{CH}_{2} \mathrm{NH}\right), 5.48\left(\mathrm{~d}, 1 \mathrm{H},{ }^{3} J_{1^{\prime}-2^{\prime}}=9.2 \mathrm{~Hz}, 1 \mathrm{H}, \mathrm{H}_{1^{\prime}}\right), 7.83-7.91(\mathrm{~m}, 4 \mathrm{H}$, $\mathrm{Ph} \mathrm{H}), 8.12(\mathrm{~s}, 1 \mathrm{H}$, triazole $\mathrm{H}) ;{ }^{13} \mathrm{C}\left\{{ }^{1} \mathrm{H}\right\}$ NMR $\left(100 \mathrm{MHz}, 2 \% \mathrm{D}_{2} \mathrm{O}\right.$ in DMSO-d $\left.d_{6}\right): \delta 35.20$ $\left(\mathrm{CH}_{2} \mathrm{NH}\right), 68.55\left(\mathrm{C}_{4}\right), 69.68\left(\mathrm{C}_{5^{\prime}}\right), 69.84\left(\mathrm{C}_{2}{ }^{\prime}\right), 72.77\left(\mathrm{C}_{3}\right), 88.55\left(\mathrm{C}_{1}\right)$ ), 123.02 (triazole $\left.\mathrm{CH}\right)$, 125.51 ( $\mathrm{Ph} \mathrm{CH}), 128.53(\mathrm{Ph} \mathrm{CH}), 137.67(\mathrm{Ph} \mathrm{C}), 144.64$ (triazole $\mathrm{C}$ or $\mathrm{Ph} \mathrm{C}), 145.83$ (triazole $\mathrm{C}$ or $\mathrm{Ph} \mathrm{C}), 169.18(\mathrm{C}=\mathrm{O})$. HRMS (ESI) calcd for $\mathrm{C}_{15} \mathrm{H}_{18} \mathrm{~N}_{5} \mathrm{O}_{7} \mathrm{~S}^{-}$: 412.093242. Found: 412.092448 .

4-(\{[4-(Aminosulfonyl)benzoyl]amino\} methyl-1-(2'-acetamido-2'-deoxy-3',4',6'-tri-Oacetyl-B-D-glucopyranosyl)-1-H-1,2,3-triazole (2d). The title compound was prepared 
according to General Procedure 1 and isolated as white solid (149 mg, $0.24 \mathrm{mmol}, 91 \%$ ). $R_{f}$ 0.32 (1:9 $\mathrm{CH}_{3} \mathrm{OH}:$ EtOAc); mp $231-232{ }^{\circ} \mathrm{C}$ (decomp). ${ }^{1} \mathrm{H}$ NMR (400 MHz, DMSO- $\left.d_{6}\right): \delta$ 1.55 (s, 3H, NHAc $\mathrm{CH}_{3}$ ), 1.91 (s, 3H, OAc $\mathrm{CH}_{3}$ ), 1.95 (s, 3H, OAc, $\mathrm{CH}_{3}$ ), 1.97 (s, 3H, OAc $\left.\mathrm{CH}_{3}\right), 4.00\left(\mathrm{dd},{ }^{2} J_{6^{\prime}-6^{\prime}},=12.4 \mathrm{~Hz},{ }^{3} J_{6^{\prime}-5^{\prime}}=2 \mathrm{~Hz}, 1 \mathrm{H}, \mathrm{H}_{6^{\prime}}\right), 4.10\left(\mathrm{dd},{ }^{2} J_{6^{\prime}-6^{\prime}}=12 \mathrm{~Hz},{ }^{3} J_{6^{\prime}-5^{\prime}}=4.8\right.$ $\left.\mathrm{Hz}, 1 \mathrm{H}, \mathrm{H}_{6^{\prime}}{ }^{\prime}\right), 4.18\left(\mathrm{ddd},{ }^{3} J_{5^{\prime}-4^{\prime}}=7.2 \mathrm{~Hz},{ }^{3} J_{5^{\prime}-6^{\prime}}=4.8 \mathrm{~Hz},{ }^{3} J_{5^{\prime}-6^{\prime}}=2 \mathrm{~Hz}, 1 \mathrm{H}, \mathrm{H}_{5^{\prime}}\right), 4.50\left(\mathrm{~d},{ }^{3} J_{\mathrm{CH}-}\right.$ $\left.\mathrm{NH}=6.0 \mathrm{~Hz}, 2 \mathrm{H}, \mathrm{CH}_{2} \mathrm{NH}\right), 4.51-4.58\left(\mathrm{~m}, 1 \mathrm{H}, \mathrm{H}_{2}{ }^{\prime}\right), 5.02-5.08\left(\mathrm{~m}, 1 \mathrm{H}, \mathrm{H}_{4}{ }^{\prime}\right), 5.29-5.34(\mathrm{~m}$, $\left.1 \mathrm{H}, \mathrm{H}_{3^{\prime}}\right), 6.07\left(\mathrm{~d},{ }^{3} J_{1^{\prime}-2^{\prime}}=10.0 \mathrm{~Hz}, 1 \mathrm{H}, \mathrm{H}_{1^{\prime}}\right), 7.44\left(\mathrm{~s}, 2 \mathrm{H}, \mathrm{SO}_{2} \mathrm{NH}_{2}\right), 7.85-8.00(\mathrm{~m}, \mathrm{Ph} \mathrm{H})$, $8.03\left(\mathrm{~d},{ }^{3} J_{\mathrm{NH}-2},=9.2 \mathrm{~Hz}, 1 \mathrm{H}, \mathrm{NHAc} \mathrm{CONH}\right), 8.13(\mathrm{~s}$, triazole $\mathrm{H}), 9.20\left(\mathrm{t},{ }^{3} \mathrm{~J}_{\mathrm{NH}-\mathrm{CH}}=6.0 \mathrm{~Hz}\right.$, $\left.1 \mathrm{H}, \mathrm{CH}_{2} \mathrm{NH}\right) ;{ }^{13} \mathrm{C}\left\{{ }^{1} \mathrm{H}\right\}$ NMR $\left(100 \mathrm{MHz}, \mathrm{DMSO}-d_{6}\right): \delta 20.96\left(\mathrm{OAc} \mathrm{CH}_{3}\right), 21.09\left(\mathrm{OAc} \mathrm{CH}_{3}\right)$, $21.20\left(\mathrm{OAc} \mathrm{CH}_{3}\right), 23.02\left(\mathrm{OAc}_{\mathrm{CH}}\right), 35.49\left(\mathrm{CH}_{2} \mathrm{NH}\right), 52.69\left(\mathrm{C}_{2},\right), 62.50\left(\mathrm{C}_{6}\right), 68.67\left(\mathrm{C}_{3}\right)$, $73.07\left(\mathrm{C}_{4}{ }^{\prime}\right), 74.05\left(\mathrm{C}_{5}{ }^{\prime}\right), 85.29\left(\mathrm{C}_{1}{ }^{\prime}\right), 122.45($ triazole $\mathrm{CH}), 126.70(\mathrm{Ph} \mathrm{CH}), 128.67(\mathrm{Ph} \mathrm{CH})$, 137.70 (Ph C), 145.80 (triazole $\mathrm{C}$ or $\mathrm{Ph} \mathrm{C}) 147.02$ (triazole $\mathrm{C}$ or $\mathrm{Ph} \mathrm{C}), 165.87(\mathrm{C}=\mathrm{O}), 170.01$ $(\mathrm{C}=\mathrm{O}), 170.11(\mathrm{C}=\mathrm{O}), 170.27(\mathrm{C}=\mathrm{O}), 170.72(\mathrm{C}=\mathrm{O})$. HRMS (ESI) calcd. for $\mathrm{C}_{24} \mathrm{H}_{29} \mathrm{~N}_{6} \mathrm{O}_{11} \mathrm{~S}^{-}$: 609.16205. Found: 609.162988. Anal. $\left(\mathrm{C}_{24} \mathrm{H}_{30} \mathrm{~N}_{6} \mathrm{O}_{11} \mathrm{~S}\right) \mathrm{C}, \mathrm{H} ; \mathrm{N}$ : calcd, 13.76; found, 12.87.

\section{4-(\{[4-(Aminosulfonyl)benzoyl]amino\} methyl-1-(2'-acetamido-2'-deoxy- $\beta$-D-}

glucopyranosyl)-1-H-1,2,3-triazole (2d'). The title compound was prepared to General Procedure 2 and isolated as white solid (79 mg, $0.16 \mathrm{mmol}, \sim 100 \%) . R_{f} 0.09$ (1:9 $\left.\mathrm{H}_{2} \mathrm{O}: \mathrm{CH}_{3} \mathrm{CN}\right)$; mp $214-215{ }^{\circ} \mathrm{C} .{ }^{1} \mathrm{H}$ NMR (400 MHz, DMSO-d 6 ): $\delta 1.62\left(\mathrm{~s}, 3 \mathrm{H}, \mathrm{NHAc} \mathrm{CH}_{3}\right)$, $3.47-3.52\left(\mathrm{~m}, 1 \mathrm{H}, \mathrm{H}_{3}{ }^{\prime}\right), 3.64-3.68\left(\mathrm{~m}, 1 \mathrm{H}, \mathrm{H}_{4}{ }^{\prime}\right), 3.97-4.05\left(\mathrm{~m}, 1 \mathrm{H}, \mathrm{H}_{2}\right), 4.48\left(\mathrm{~d},{ }^{3} J_{\mathrm{CH}-\mathrm{NH}}=\right.$ $\left.5.8 \mathrm{~Hz}, 2 \mathrm{H}, \mathrm{CH}_{2} \mathrm{NH}\right), 4.58-4.61\left(\mathrm{~m}, 1 \mathrm{H}, \mathrm{H}_{6}{ }^{\prime}\right), 5.21-5.24\left(\mathrm{~m}, 2 \mathrm{H}, \mathrm{H}_{3}, \mathrm{H}_{4}{ }^{\prime}\right), 5.66\left(\mathrm{~d}, 1 \mathrm{H},{ }^{3} J_{1}\right.$ '$\left.2^{2}=9.6 \mathrm{~Hz}, 1 \mathrm{H}, \mathrm{H}_{1}\right), 7.44\left(\right.$ br s, $\left.2 \mathrm{H}, \mathrm{SO}_{2} \mathrm{NH}_{2}\right), 7.82\left(\mathrm{~d},{ }^{3} J_{\mathrm{NH}-2},=8.8 \mathrm{~Hz}, 1 \mathrm{H}\right.$, NHAc $\left.\mathrm{NH}\right)$, $7.85-8.00(\mathrm{~m}, 4 \mathrm{H}, \mathrm{Ph}), 7.97(\mathrm{~s}, 1 \mathrm{H}$, triazole $1 \mathrm{H}), 9.19\left(\mathrm{t},{ }^{3} \mathrm{~J}_{\mathrm{NH}-\mathrm{CH}}=6.0 \mathrm{~Hz}, 1 \mathrm{H}, \mathrm{CH}_{2} \mathrm{NH}\right) ;{ }^{13} \mathrm{C}$ $\left\{{ }^{1} \mathrm{H}\right\}$ NMR (100 MHz, 2\% $\mathrm{D}_{2} \mathrm{O}$ in DMSO-d $\left.)_{6}\right): \delta 23.30\left(\mathrm{NHAc} \mathrm{CH}_{3}\right), 35.40\left(\mathrm{CH}_{2} \mathrm{NH}\right), 55.00$ $\left(\mathrm{C}_{2},\right), 61.81\left(\mathrm{C}_{6^{\prime}}\right), 70.44\left(\mathrm{C}_{4}{ }^{\prime}\right), 74.46\left(\mathrm{C}_{3}{ }^{\prime}\right), 80.63\left(\mathrm{C}_{5},\right), 86.59\left(\mathrm{C}_{1}{ }^{\prime}\right), 122.22$ (triazole $\left.\mathrm{CH}\right)$, 
126.32 (Ph CH), $128.66(\mathrm{Ph} \mathrm{CH}), 137.68(\mathrm{Ph} \mathrm{C}), 145.26$ (triazole $\mathrm{C}$ or $\mathrm{Ph} \mathrm{C}), 146.87$ (triazole $\mathrm{C}$ or $\mathrm{Ph} \mathrm{C}), 166.10(\mathrm{O}=\mathrm{C}), 169.982(\mathrm{C}=\mathrm{O})$. HRMS (ESI) calcd for $\mathrm{C}_{18} \mathrm{H}_{23} \mathrm{~N}_{6} \mathrm{O}_{8} \mathrm{~S}^{-}$: 483.130356. Found: 483.129389.

4-(\{[4-(Aminosulfonyl)benzoyl]amino\} methyl-1-(2',3',4'-tri-O-acetyl- $\beta$-D-glucuronic acid methyl ester)-1-H-1,2,3-triazole (2e). The title compound was prepared according to General Procedure 1 and isolated as white solid (153 mg, $0.26 \mathrm{mmol}, 92 \%) . R_{f} 0.12$ (1:9 Hexanes:EtOAc); mp $221-222{ }^{\circ} \mathrm{C}$ (decomp). ${ }^{1} \mathrm{H}$ NMR (400 MHz, DMSO-d $\left.)_{6}\right): \delta 1.76$ (s, 3H, OAc), 1.95 (s, 3H, OAc), $1.98(\mathrm{~s}, 3 \mathrm{H}, \mathrm{OAc}), 3.59\left(\mathrm{~s}, 3 \mathrm{H}, \mathrm{OCH}_{3}\right), 4.51\left(\mathrm{~d},{ }^{3} J_{\mathrm{CH}-\mathrm{NH}}=5.2 \mathrm{~Hz}\right.$, $\left.2 \mathrm{H}, \mathrm{CH}_{2} \mathrm{NH}\right), 4.76\left(\mathrm{~d},{ }^{3} \mathrm{~J}_{5^{\prime}-4^{\prime}}=10.0 \mathrm{~Hz}, 1 \mathrm{H}, \mathrm{H}_{5}{ }^{\prime}\right), 5.19-5.24\left(\mathrm{~m}, 1 \mathrm{H}, \mathrm{H}_{4}{ }^{\prime}\right), 5.44-5.59(\mathrm{~m}, 1 \mathrm{H}$, $\left.\mathrm{H}_{3^{\prime}}\right), 5.72-5.76\left(\mathrm{~m}, 1 \mathrm{H}, \mathrm{H}_{2}{ }^{\prime}\right), 6.34\left(\mathrm{~d},{ }^{3} \mathrm{~J}_{1^{\prime}-2}\right.$, $\left.=9.6 \mathrm{~Hz}, 1 \mathrm{H}, \mathrm{H}_{1^{\prime}}\right), 7.45$ (br s, $\left.2 \mathrm{H}, \mathrm{SO}_{2} \mathrm{NH}_{2}\right)$, $7.86-8.01(\mathrm{~m}, 4 \mathrm{H}, \mathrm{Ph} \mathrm{H}), 8.35(\mathrm{~s}, 1 \mathrm{H}$, triazole $\mathrm{H}), 9.23\left(\mathrm{t},{ }^{3} J_{\mathrm{NH}-\mathrm{CH}}=5.6 \mathrm{~Hz}, 1 \mathrm{H}, \mathrm{NHCH}_{2}\right)$; ${ }^{13} \mathrm{C}\left\{{ }^{1} \mathrm{H}\right\}$ NMR $\left(100 \mathrm{MHz}, \mathrm{DMSO}-d_{6}\right): \delta 20.6\left(\mathrm{OAc} \mathrm{CH}_{3}\right), 20.9\left(\mathrm{OAc} \mathrm{CH}_{3}\right), 21.0\left(\mathrm{OAc} \mathrm{CH}_{3}\right)$, $35.5\left(\mathrm{CH}_{2} \mathrm{NH}\right), 53.3\left(\mathrm{CO}_{2} \mathrm{CH}_{3}\right), 69.0\left(\mathrm{C}_{4}\right), 70.4\left(\mathrm{C}_{2}\right), 72.3\left(\mathrm{C}_{3}\right), 73.5\left(\mathrm{C}_{5}\right), 84.4\left(\mathrm{C}_{1}\right), 122.9$ (triazole $\mathrm{CH}), 126.3(\mathrm{Ph} \mathrm{CH}), 128.7(\mathrm{Ph} \mathrm{CH}), 137.6(\mathrm{Ph} \mathrm{C}), 146.4$ (triazole $\mathrm{C}$ or $\mathrm{Ph} \mathrm{C}), 147.1$ (triazole $\mathrm{C}$ or $\mathrm{Ph} \mathrm{C}), 165.9(\mathrm{C}=\mathrm{O}), 167.3(\mathrm{C}=\mathrm{O}), 169.1(\mathrm{C}=\mathrm{O}), 170.0(\mathrm{C}=\mathrm{O}), 170.2(\mathrm{C}=\mathrm{O})$.

HRMS (ESI) calcd for $\mathrm{C}_{23} \mathrm{H}_{26} \mathrm{~N}_{5} \mathrm{O}_{12} \mathrm{~S}^{-}$: 596.130416. Found: 596.132499. Anal. $\left(\mathrm{C}_{23} \mathrm{H}_{27} \mathrm{~N}_{5} \mathrm{O}_{12} \mathrm{~S}\right) \mathrm{C}, \mathrm{H}, \mathrm{N}$.

\section{4-(\{[4-(Aminosulfonyl)benzoyl]amino\}methyl-1-( $\beta$-D-glucuronic acid methyl ester)-1-H-}

1,2,3-triazole (2⿺') $)$. The title compound was prepared according to General Procedure 2 and isolated as clear gum (79 mg, $0.17 \mathrm{mmol}, \sim 100 \%) . R_{f} 0.13$ (1:9 MeOH:EtOAc). ${ }^{1} \mathrm{H}$ NMR (400 MHz, 2\% $\mathrm{D}_{2} \mathrm{O}$ in DMSO-d $\left.\mathrm{D}_{6}\right): \delta 3.40-3.49\left(\mathrm{~m}, 2 \mathrm{H}, \mathrm{H}_{3}, \mathrm{H}_{4}\right.$ ) $), 3.61\left(\mathrm{~s}, 3 \mathrm{H}, \mathrm{COCH}_{3}\right), 3.82$ $-3.86\left(\mathrm{~m}, 1 \mathrm{H}, \mathrm{H}_{2^{\prime}}\right), 4.08\left(\mathrm{~d},{ }^{3} J_{5^{\prime}-4^{\prime}}=9.6 \mathrm{~Hz}, 1 \mathrm{H}, \mathrm{H}_{5^{\prime}}\right), 4.51\left(\mathrm{~s}, 2 \mathrm{H}, \mathrm{CH}_{2} \mathrm{NH}\right), 5.66\left(\mathrm{~d},{ }^{3} J_{1^{\prime}-2^{\prime}}=\right.$ $\left.9.6 \mathrm{~Hz}, 1 \mathrm{H}, \mathrm{H}_{1}\right), 7.85-8.00(\mathrm{~m}, 4 \mathrm{H}, \mathrm{Ph}), 8.16(\mathrm{~s}, 1 \mathrm{H}$, triazole $\mathrm{CH}) ;{ }^{13} \mathrm{C}\left\{{ }^{1} \mathrm{H}\right\} \mathrm{NMR}(100$ 
MHz, $2 \% \mathrm{D}_{2} \mathrm{O}$ in DMSO- $\left.d_{6}\right): \delta 35.43\left(\mathrm{CH}_{2} \mathrm{NH}\right), 52.74\left(\mathrm{COCH}_{3}\right), 71.63\left(\mathrm{C}_{3}{ }^{\prime}\right), 71.94\left(\mathrm{C}_{4}{ }^{\prime}\right)$, $76.63\left(\mathrm{C}_{2}\right), 78.02\left(\mathrm{C}_{5}\right), 87.66\left(\mathrm{C}_{1}\right), 123.08$ (triazole $\left.\mathrm{CH}\right), 126.31(\mathrm{Ph} \mathrm{CH}), 128.69(\mathrm{Ph} \mathrm{CH})$, 137.64 (Ph C), 145.52 (triazole C or Ph C), 146.89 (triazole $\mathrm{C}$ or $\mathrm{Ph} \mathrm{C}), 165.85(\mathrm{C}=\mathrm{O}), 169.37$ $(\mathrm{C}=\mathrm{O})$. HRMS (ESI) calcd for $\mathrm{C}_{17} \mathrm{H}_{20} \mathrm{~N}_{5} \mathrm{O}_{9} \mathrm{~S}^{-}: 470.098722$. Found: 470.097959 .

\section{4-(\{[4-(Aminosulfonyl)benzoyl]amino $\}$ methyl-1-(hepta-O-acetyl- $\beta$-D-maltosyl)-1- $H-1,2,3-$}

triazole (2f). The title compound was prepared according to General Procedure 1 and isolated as pale yellow foam (424 mg, $0.47 \mathrm{mmol}, 78 \%) . R_{f} 0.23\left(2: 8\right.$ Hexanes:EtOAc). ${ }^{1} \mathrm{H}$ NMR (400 MHz, DMSO-d ${ }_{6}$ ): $\delta 1.72\left(\mathrm{~s}, 3 \mathrm{H}, \mathrm{OAc} \mathrm{CH}_{3}\right), 1.92\left(\mathrm{~s}, 3 \mathrm{H}, \mathrm{OAc}_{\mathrm{CH}}\right.$ ), $1.93(\mathrm{~s}, 3 \mathrm{H}$, OAc $\left.\mathrm{CH}_{3}\right), 1.96\left(\mathrm{~s}, 3 \mathrm{H}, \mathrm{OAc} \mathrm{CH}_{3}\right), 1.97$ (s, 3H, OAc $\mathrm{CH}_{3}$ ), 1.99 (s, 3H, OAc $\left.\mathrm{CH}_{3}\right), 2.00$ (s,

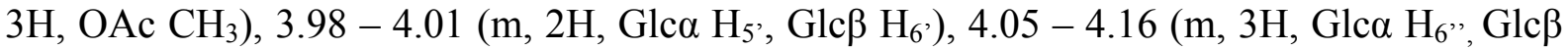
$\left.\mathrm{H}_{4^{\prime}}, \mathrm{Glc} \beta \mathrm{H}_{6^{\prime}}\right), 4.29\left(\mathrm{ddd},{ }^{3} J_{5^{\prime}-4^{\prime}}=9.6 \mathrm{~Hz},{ }^{3} J_{5^{\prime}-6^{\prime}}=5.6 \mathrm{~Hz},{ }^{3} J_{5^{\prime}-6^{\prime}},=2.4 \mathrm{~Hz}, 1 \mathrm{H}, \mathrm{Glc} \beta \mathrm{H}_{5^{\prime}}\right.$ ), 4.40 $\left(\mathrm{dd},{ }^{2} J_{6^{\prime}-6^{\prime}},=12.4 \mathrm{~Hz},{ }^{3} J_{6^{\prime}-5},=2.4 \mathrm{~Hz}, 1 \mathrm{H}, \mathrm{Glc} \beta \mathrm{H}_{6^{\prime}}\right), 4.50\left(\mathrm{~d},{ }^{3} J_{\mathrm{CH}-\mathrm{NH}}=5.6 \mathrm{~Hz}, 2 \mathrm{H}, \mathrm{CH}_{2} \mathrm{NH}\right)$,

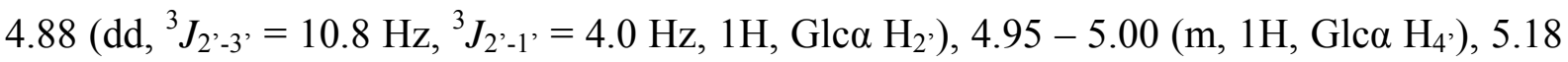
$-5.23\left(\mathrm{~m}, 1 \mathrm{H}, \mathrm{Glc} \alpha \mathrm{H}_{3}\right), 5.32\left(\mathrm{~d},{ }^{3} J_{1^{\prime}-2}{ }^{\prime}=4.0 \mathrm{~Hz}, 1 \mathrm{H}, \mathrm{Glc} \alpha \mathrm{H}_{1^{\prime}}\right), 5.47-5.57(\mathrm{~m}, 2 \mathrm{H}, \mathrm{Glc} \beta$ $\left.\mathrm{H}_{2}, \mathrm{Glc} \beta \mathrm{H}_{3}{ }^{\prime}\right), 6.25\left(\mathrm{~d},{ }^{3} J_{1^{\prime}-2},=9.2 \mathrm{~Hz}, 1 \mathrm{H}, \mathrm{Glc} \beta \mathrm{H}_{1}{ }^{\prime}\right), 7.45$ (br s, $\left.2 \mathrm{H}, \mathrm{SO}_{2} \mathrm{NH}_{2}\right), 7.86-7.99$ $(\mathrm{m}, 4 \mathrm{H}, \mathrm{Ph}), 8.16(\mathrm{~s}, 1 \mathrm{H}$, triazole $\mathrm{CH}), 9.21\left(\mathrm{t},{ }^{3} \mathrm{~J}_{\mathrm{NH}-\mathrm{CH}}=5.6 \mathrm{~Hz}, 1 \mathrm{H}, \mathrm{CH}_{2} \mathrm{NH}\right) ;{ }^{13} \mathrm{C}\left\{{ }^{1} \mathrm{H}\right\} \mathrm{NMR}$ (100 MHz, DMSO-d $\left.d_{6}\right): \delta 20.63\left(\mathrm{OAc} \mathrm{CH}_{3}\right), 20.94\left(\mathrm{OAc} \mathrm{CH}_{3}\right), 21.00\left(\mathrm{OAc} \mathrm{CH}_{3}\right), 21.10$ $\left(\mathrm{OAc} \mathrm{CH}_{3}\right), 21.20\left(\mathrm{OAc} \mathrm{CH}_{3}\right), 21.24\left(\mathrm{OAc} \mathrm{CH}_{3}\right), 35.51\left(\mathrm{CH}_{2} \mathrm{NH}\right), 62.06\left(\mathrm{Glc} \beta \mathrm{C}_{6}{ }^{\prime}\right), 63.52$

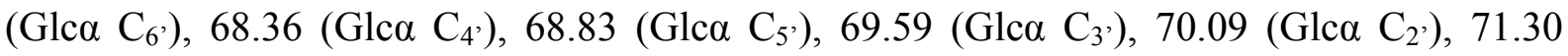

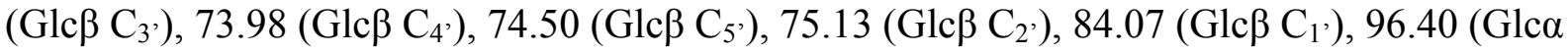
$\mathrm{C}_{1}$ ), 122.74 (triazole $\left.\mathrm{CH}\right), 126.29(\mathrm{Ph} \mathrm{CH}), 128.64(\mathrm{Ph} \mathrm{CH}), 137.64(\mathrm{Ph} \mathrm{C}), 146.19$ (triazole $\mathrm{C}$ or Ph C), 147.05 (triazole $\mathrm{C}$ or Ph C), $165.87(\mathrm{C}=\mathrm{O})$. HRMS (ESI) calcd for $\mathrm{C}_{36} \mathrm{H}_{44} \mathrm{~N}_{5} \mathrm{O}_{20} \mathrm{~S}^{-}$ : 898.230583. Found: 898.228045. Anal $\left(\mathrm{C}_{36} \mathrm{H}_{45} \mathrm{~N}_{5} \mathrm{O}_{20} \mathrm{~S} \cdot 3 \mathrm{H}_{2} \mathrm{O}\right) \mathrm{C}, \mathrm{H}, \mathrm{N}, \mathrm{S}$. 
The title compound was prepared according to General Procedure 2 and isolated as pale yellow foam $(134 \mathrm{mg}, 0.22 \mathrm{mmol} \sim 100 \%) . R_{f} 0.10\left(1: 9 \mathrm{H}_{2} \mathrm{O}: \mathrm{CH}_{3} \mathrm{CN}\right) .{ }^{1} \mathrm{H}$ NMR $(400 \mathrm{MHz}$, $\left.\mathrm{D}_{2} \mathrm{O}\right): \delta 3.27-3.32\left(\mathrm{~m}, 1 \mathrm{H}, \mathrm{Glc} \alpha \mathrm{H}_{4}\right.$, or $\left.\mathrm{Glc} \beta \mathrm{H}_{4}\right), 3.46\left(\mathrm{dd},{ }^{3} J_{2^{\prime}-3^{\prime}}=10.0 \mathrm{~Hz},{ }^{3} J_{2^{\prime}-1},=4.0 \mathrm{~Hz}\right.$, Glc $\left.\alpha \mathrm{H}_{2}{ }^{\prime}\right), 3.55-3.79\left(\mathrm{~m}, 8 \mathrm{H}\right.$, Glc $\alpha \mathrm{H}_{3}{ }^{\prime}$, Glc $\alpha \mathrm{H}_{5}{ }^{\prime}$, Glc $\alpha \mathrm{H}_{6 /} \mathrm{H}_{6}$ ', and Glc $\beta \mathrm{H}_{3}{ }^{\prime}$, Glc $\beta \mathrm{H}_{5}{ }^{\prime}$, Glc $\left.\beta \mathrm{H}_{6}{ }^{\prime} / \mathrm{H}_{6}{ }^{\prime}\right), 3.83-3.87$ (m, $\left.1 \mathrm{H}, \mathrm{Glc} \beta \mathrm{H}_{3}{ }^{\prime}\right), 3.88$ - 3.92 (m, $\left.1 \mathrm{H}, \mathrm{Glc} \beta \mathrm{H}_{2}{ }^{\prime}\right) 4.58$ (s, 2H,

$\left.\mathrm{CH}_{2} \mathrm{NH}\right), 5.33\left(\mathrm{~d},{ }^{3} J_{1^{\prime}-2^{\prime}}=4.0 \mathrm{~Hz}, 1 \mathrm{H}, \mathrm{Glc} \alpha \mathrm{H}_{1}{ }^{\prime}\right), 5.62\left(\mathrm{~d},{ }^{3} J_{1^{\prime}-2^{\prime}}=9.2 \mathrm{~Hz} 1 \mathrm{H}, \mathrm{Glc} \beta \mathrm{H}_{1}{ }^{\prime}\right), 7.77$

$-7.86(\mathrm{~m}, 4 \mathrm{H}, \mathrm{Ph}), 8.07(\mathrm{~s}, 1 \mathrm{H}$, triazole $\mathrm{CH}) ;{ }^{13} \mathrm{C}\left\{{ }^{1} \mathrm{H}\right\}$ NMR $\left(100 \mathrm{MHz}, \mathrm{D}_{2} \mathrm{O}\right): \delta 35.18$ $\left(\mathrm{CH}_{2} \mathrm{NH}\right), 60.58\left(\mathrm{Glc} \mathrm{C}_{6}\right.$, or $\left.\mathrm{Glc} \beta \mathrm{C}_{6}{ }\right), 60.59\left(\mathrm{Glc} \mathrm{C}_{6}\right.$, or $\mathrm{Glc} \beta \mathrm{C}_{6}$ ) $), 69.47,71.84,72.27$, 72.91, 72.99, 75.97, 76.50, 77.63 (Glc $\alpha \mathrm{C}_{2}$, Glc $\alpha \mathrm{C}_{3}$, Glc $\alpha \mathrm{C}_{4}$, Glc $\alpha \mathrm{C}_{5}$, and Glc $\beta \mathrm{C}_{2}$, Glc $\beta$ $\mathrm{C}_{3}$, Glc $\beta \mathrm{C}_{4}, \mathrm{Glc} \mathrm{C}_{5}$ ), $87.42\left(\mathrm{Glc} \mathrm{C}_{1}\right.$ ) $), 99.83\left(\mathrm{Glc} \mathrm{C}_{1}\right.$ ) $), 123.32$ (triazole $\left.\mathrm{CH}\right), 126.33(\mathrm{Ph}$ $\mathrm{CH}), 128.36(\mathrm{Ph} \mathrm{CH}), 137.39$ ( $\mathrm{Ph} \mathrm{C}), 144.70$ (triazole $\mathrm{C}$ or $\mathrm{Ph} \mathrm{C}), 144.97$ (triazole $\mathrm{C}$ or $\mathrm{Ph} \mathrm{C}$ ), 169.32 $(\mathrm{C}=\mathrm{O})$. HRMS (ESI) calcd for $\mathrm{C}_{22} \mathrm{H}_{30} \mathrm{~N}_{5} \mathrm{O}_{13} \mathrm{~S}^{-}: 604.15663$. Found: 604.155698 .

\section{Methyl 2',3',4'-tri-O-acetyl-6-[4-(\{[aminosulfonyl)benzoyl]amino\}methyl)-1H-1,2,3-}

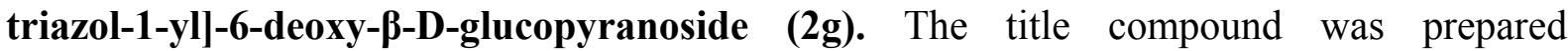
according to General Procedure 1 and isolated as white foam $(278 \mathrm{mg}, 0.48 \mathrm{mmol}, 82 \%)$. $R_{f}$ $0.1\left(100 \%\right.$ EtOAc). ${ }^{1} \mathrm{H}$ NMR (400 MHz, DMSO- $\left.d_{6}\right): \delta 1.92\left(\mathrm{~s}, 3 \mathrm{H}, \mathrm{OAc}_{\mathrm{CH}}\right), 1.96(\mathrm{~s}, 3 \mathrm{H}$, OAc $\left.\mathrm{CH}_{3}\right), 1.98\left(\mathrm{~s}, 3 \mathrm{H}, \mathrm{OAc} \mathrm{CH}_{3}\right), 3.03\left(\mathrm{~s}, 3 \mathrm{H}, \mathrm{OCH}_{3}\right), 4.11\left(\mathrm{~m}, 1 \mathrm{H}, \mathrm{H}_{5}\right), 4.44-4.49(\mathrm{~m}, 2 \mathrm{H}$, $\left.\mathrm{H}_{6}, \mathrm{CH}_{2} \mathrm{NH}\right), 4.55\left(\mathrm{dd},{ }^{2} J_{6^{\prime}-6^{\prime}}=14.4 \mathrm{~Hz},{ }^{3} J_{6^{\prime}-5},=2.8 \mathrm{~Hz}, 1 \mathrm{H}, \mathrm{H}_{6^{\prime}}\right), 4.78-4.82\left(\mathrm{~m}, 2 \mathrm{H}, \mathrm{H}_{1^{\prime}}\right.$, $\left.\mathrm{H}_{2}{ }^{\prime}\right), 4.83-4.88\left(\mathrm{~m}, 1 \mathrm{H}, \mathrm{H}_{4}{ }^{\prime}\right), 5.21-5.26\left(\mathrm{~m}, 1 \mathrm{H}, \mathrm{H}_{3}{ }^{\prime}\right), 7.44\left(\right.$ br s, $\left.2 \mathrm{H}, \mathrm{SO}_{2} \mathrm{NH}_{2}\right), 7.85-7.98$ $(\mathrm{m}, 4 \mathrm{H}, \mathrm{Ph}), 7.97(\mathrm{~s}, 1 \mathrm{H}$, triazole $\mathrm{CH}), 9.19\left(\mathrm{t},{ }^{3} J_{\mathrm{NH}-\mathrm{CH}}=5.6 \mathrm{~Hz}, 1 \mathrm{H}, \mathrm{CH}_{2} \mathrm{NH}\right) ;{ }^{13} \mathrm{C} \mathrm{NMR}\left\{{ }^{1} \mathrm{H}\right\}$ $\left(100 \mathrm{MHz}, \mathrm{DMSO}-d_{6}\right): \delta 20.96\left(\mathrm{OAc} \mathrm{CH}_{3}\right), 21.03\left(\mathrm{OAc} \mathrm{CH}_{3}\right), 21.13\left(\mathrm{OAc} \mathrm{CH}_{3}\right), 35.55$ $\left(\mathrm{CH}_{2} \mathrm{NH}\right), 50.40\left(\mathrm{C}_{6},\right), 55.28\left(\mathrm{OCH}_{3}\right), 68.00\left(\mathrm{C}_{5},\right), 70.12\left(\mathrm{C}_{1}\right.$, or $\left.\mathrm{C}_{2},\right), 70.17\left(\mathrm{C}_{1}\right.$, or $\left.\mathrm{C}_{2},\right), 70.33$ $\left(\mathrm{C}_{3}{ }^{\prime}\right), 96.57\left(\mathrm{C}_{1}{ }^{\prime}\right), 124.54$ (triazole $\left.\mathrm{CH}\right), 126.31(\mathrm{Ph} \mathrm{CH}), 128.58(\mathrm{Ph} \mathrm{CH}), 137.68(\mathrm{Ph} \mathrm{C})$, 
145.52 (triazole $\mathrm{C}$ or $\mathrm{Ph} \mathrm{C}) 146.95$ (triazole $\mathrm{C}$ or $\mathrm{Ph} \mathrm{C}), 165.89(\mathrm{C}=\mathrm{O}), 170.07(\mathrm{C}=\mathrm{O}), 170.34$ $(\mathrm{C}=\mathrm{O}), \quad 170.41 \quad(\mathrm{C}=\mathrm{O})$. HRMS (ESI) calcd for $\mathrm{C}_{23} \mathrm{H}_{27} \mathrm{~N}_{4} \mathrm{O}_{12} \mathrm{~S}^{-}$: 582.151151. Found: 582.150947. Anal $\left(\mathrm{C}_{23} \mathrm{H}_{29} \mathrm{~N}_{5} \mathrm{O}_{11} \mathrm{~S} \cdot \mathrm{H}_{2} \mathrm{O}\right) \mathrm{C}, \mathrm{H}, \mathrm{N}$.

\section{Methyl 6-[4-(\{[aminosulfonyl)benzoyl]amino\}methyl)-1H-1,2,3-triazol-1-yl]-6-deoxy- $\beta$ -}

D-glucopyranoside (2g'). The title compound was prepared according to General Procedure 1 and isolated as pale yellow foam (76 mg, $0.17 \mathrm{mmol}, 97 \%) . R_{f} 0.21$ (1:9 $\left.\mathrm{CH}_{3} \mathrm{OH}: \mathrm{EtOAc}\right)$. ${ }^{1} \mathrm{H}$ NMR (400 MHz, 2\% D $2 \mathrm{O}$ in DMSO-d $): \delta 2.87\left(\mathrm{~s}, 3 \mathrm{H}, \mathrm{OCH}_{3}\right), 2.96\left(\mathrm{dd},{ }^{3} J_{4}{ }^{\prime}-5,=10.4 \mathrm{~Hz}\right.$, $\left.{ }^{3} J_{4^{\prime}-3^{\prime}}=8.8 \mathrm{~Hz}, 1 \mathrm{H}, \mathrm{H}_{4^{\prime}}\right), 3.14\left(\mathrm{dd},{ }^{3} J_{2^{\prime}-3^{\prime}}=9.6 \mathrm{~Hz},{ }^{3} J_{2^{\prime}-1},=3.6 \mathrm{~Hz}, 1 \mathrm{H}, \mathrm{H}_{2}\right), 3.31-3.36(\mathrm{~m}$, $\left.1 \mathrm{H}, \mathrm{H}_{3^{\prime}}\right), 3.59-3.65\left(\mathrm{~m}, 1 \mathrm{H}, \mathrm{H}_{5^{\prime}}\right), 4.33\left(\mathrm{dd},{ }^{2} J_{6^{\prime}-6^{\prime}},=14.0 \mathrm{~Hz},{ }^{3} J_{6^{\prime}-5}=9.2 \mathrm{~Hz}, 1 \mathrm{H}, \mathrm{H}_{6^{\prime}}\right), 4.42$ $\left(\mathrm{d}, 1 \mathrm{H},{ }^{3} J_{1^{\prime}-2^{\prime}}=3.6 \mathrm{~Hz}, 1 \mathrm{H}, \mathrm{H}_{1}\right.$ ) $), 4.47-4.48\left(\mathrm{~m}, 2 \mathrm{H}, \mathrm{CH}_{2} \mathrm{NH}\right), 4.65\left(\mathrm{dd},{ }^{2} \mathrm{~J}_{6}{ }^{\prime}-6^{\prime}=14.0 \mathrm{~Hz}, 1 \mathrm{H}\right.$, $\left.\mathrm{H}_{6}{ }^{\prime}\right), 7.85(\mathrm{~s}, 1 \mathrm{H}$, triazole $\mathrm{CH}), 7.86-7.97(\mathrm{~m}, 4 \mathrm{H}, \mathrm{Ph}), 9.18\left(\mathrm{t},{ }^{3} J_{\mathrm{NH}-\mathrm{CH}}=5.2 \mathrm{~Hz}, 1 \mathrm{H}\right.$ $\left.\mathrm{CH}_{2} \mathrm{NH}\right) ;{ }^{13} \mathrm{C}$ NMR $\left\{{ }^{1} \mathrm{H}\right\}\left(100 \mathrm{MHz}, 2 \% \mathrm{D}_{2} \mathrm{O}\right.$ in DMSO-d $\left.d_{6}\right): \delta 35.45\left(\mathrm{CH}_{2} \mathrm{NH}\right), 51.50\left(\mathrm{C}_{6}{ }^{\prime}\right)$, $54.87\left(\mathrm{OCH}_{3}\right), 71.07\left(\mathrm{C}_{5},\right), 72.12\left(\mathrm{C}_{4}\right.$, or $\left.\mathrm{C}_{2},\right), 72.21\left(\mathrm{C}_{4}\right.$, or $\left.\mathrm{C}_{2},\right), 73.53\left(\mathrm{C}_{3},\right), 100.31\left(\mathrm{C}_{1},\right)$, 124.50 (triazole $\mathrm{CH}), 126.33(\mathrm{Ph} \mathrm{CH}), 128.57(\mathrm{Ph} \mathrm{CH}), 137.67(\mathrm{Ph} \mathrm{C}), 145.33$ (triazole $\mathrm{C}$ or Ph C), 146.87 (triazole $\mathrm{C}$ or $\mathrm{Ph} \mathrm{C}$ ), $165.74(\mathrm{C}=\mathrm{O})$ ). HRMS (ESI) calcd for $\mathrm{C}_{17} \mathrm{H}_{21} \mathrm{~N}_{4} \mathrm{O}_{9} \mathrm{~S}^{-}$: 456.119457. Found: 456.118334.

\section{4-(\{[4-(Aminosulfonyl)benzoyl]oxy\}methyl-1-(2'-3'-4'-6'-tetra-O-acetyl-}

glucopyranosyl)-1-H-1,2,3-triazole (3a). The title compound was prepared according to General Procedure 1 and isolated as white solid (142 mg, $0.23 \mathrm{mmol}, 86 \%) . R_{f} 0.43(2: 8$ Hexanes:EtOAc); mp $196-197{ }^{\circ} \mathrm{C} .{ }^{1} \mathrm{H}$ NMR (400 MHz, DMSO-d 6 ): $\delta 1.75$ (s, 3H, OAc $\mathrm{CH}_{3}$ ), 1.93 (s, 3H, OAc $\mathrm{CH}_{3}$ ), 1.97 (s, 3H, $\mathrm{OAc} \mathrm{CH}_{3}$ ), 2.00 (s, 3H, OAc $\mathrm{CH}_{3}$ ), 4.05 (dd, ${ }^{2} J_{6}$ '-6', $\left.=12.8 \mathrm{~Hz},{ }^{3} J_{6^{\prime}-5},=2.4 \mathrm{~Hz}, 1 \mathrm{H}, \mathrm{H}_{6}{ }^{\prime}\right), 4.10\left(\mathrm{dd},{ }^{2} J_{6^{\prime}}{ }^{\prime}-6^{\prime}=12.8 \mathrm{~Hz},{ }^{3} \mathrm{H}_{6^{\prime},-5},=5.2 \mathrm{~Hz}, 1 \mathrm{H}, \mathrm{H}_{6}{ }^{\prime}\right)$, $4.35\left(\mathrm{ddd},{ }^{3} J_{5^{\prime}-4^{\prime}}=10 \mathrm{~Hz},{ }^{3} J_{5^{\prime}-6^{\prime}},=5.2 \mathrm{~Hz},{ }^{3} J_{5^{\prime}-6^{\prime}}=2.4 \mathrm{~Hz}, 1 \mathrm{H}, \mathrm{H}_{5}\right.$ ), $5.13-5.18\left(\mathrm{~m}, 1 \mathrm{H}, \mathrm{H}_{4^{\prime}}\right)$, 
$5.40-5.46\left(\mathrm{~m}, 2 \mathrm{H}, \mathrm{CH}_{2} \mathrm{O}\right), 5.51-5.55\left(\mathrm{~m}, 1 \mathrm{H}, \mathrm{H}_{3^{\prime}}\right), 5.63-5.67\left(\mathrm{~m}, 1 \mathrm{H}, \mathrm{H}_{2}{ }^{\prime}\right), 6.35\left(\mathrm{~d},{ }^{3} J_{1^{\prime}-2^{\prime}}\right.$, $\left.=9.2 \mathrm{~Hz}, 1 \mathrm{H}, \mathrm{H}_{1}{ }^{\prime}\right), 7.54\left(\mathrm{~s}, 2 \mathrm{H}, \mathrm{SO}_{2} \mathrm{NH}_{2}\right), 7.92-8.11(\mathrm{~m}, 4 \mathrm{H}, \mathrm{Ph} \mathrm{H}), 8.57(\mathrm{~s}, 1 \mathrm{H}$, triazole $\mathrm{H})$;

${ }^{13} \mathrm{C}\left\{{ }^{1} \mathrm{H}\right\}$ NMR $\left(100 \mathrm{MHz}, \mathrm{DMSO}-d_{6}\right): \delta 20.56\left(\mathrm{OAc} \mathrm{CH}_{3}\right), 20.92\left(\mathrm{OAc} \mathrm{CH}_{3}\right), 21.06(\mathrm{OAc}$

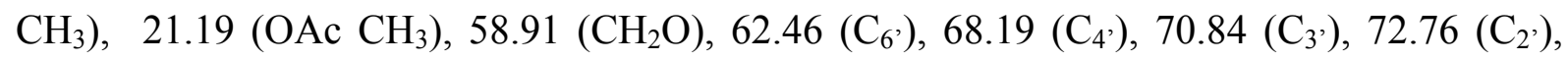
$73.97\left(\mathrm{C}_{5}{ }^{\prime}\right), 84.56\left(\mathrm{C}_{1}{ }^{\prime}\right), 124.70($ triazole $\mathrm{CH}), 126.76(\mathrm{Ph} \mathrm{CH}) 130.68(\mathrm{Ph} \mathrm{CH}) 132.69(\mathrm{Ph} \mathrm{C})$ 143.16 (triazole $\mathrm{C}$ or $\mathrm{Ph} \mathrm{C}) 148.92$ (triazole $\mathrm{C}$ or $\mathrm{Ph} \mathrm{C}), 165.09(\mathrm{C}=\mathrm{O}), 169.16(\mathrm{C}=\mathrm{O}), 170.04$ $(\mathrm{C}=\mathrm{O}), 170.22(\mathrm{C}=\mathrm{O}), 170.70(\mathrm{C}=\mathrm{O})$. HRMS (ESI) calcd for $\mathrm{C}_{24} \mathrm{H}_{27} \mathrm{~N}_{4} \mathrm{O}_{13} \mathrm{~S}^{-}: 611.130081$. Found: 611.127932. Anal $\left(\mathrm{C}_{24} \mathrm{H}_{28} \mathrm{~N}_{4} \mathrm{O}_{13} \mathrm{~S}\right) \mathrm{C}, \mathrm{H}, \mathrm{N}, \mathrm{S}$.

\section{4-(\{[4-(Aminosulfonyl)benzoyl]oxy\} methyl-1-( $\beta$-D-glucopyranosyl)-1-H-1,2,3-triazole}

(3a'). The title compound was prepared according to General Procedure 1 and isolated as white foam (82 mg, $0.19 \mathrm{mmol}, 72 \%) . R_{f} 0.10$ (3:7 EtOH: $\left.\mathrm{CHCl}_{3}\right) .{ }^{1} \mathrm{H}$ NMR $\left(400 \mathrm{MHz}, \mathrm{D}_{2} \mathrm{O}\right)$ :

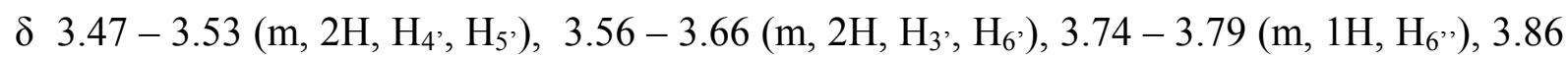
$-3.90\left(\mathrm{~m}, 1 \mathrm{H}, \mathrm{H}_{2}\right), 5.65\left(\mathrm{~d},{ }^{3} J_{1^{\prime}-2^{\prime}}=8 \mathrm{~Hz}, 1 \mathrm{H}, \mathrm{H}_{1}{ }^{\prime}\right), 7.81-8.00(\mathrm{Ph} \mathrm{H}), 8.25(\mathrm{~s}$, triazole $\mathrm{CH})$; ${ }^{13} \mathrm{C}\left\{{ }^{1} \mathrm{H}\right\}$ NMR $\left(100 \mathrm{MHz}, \mathrm{D}_{2} \mathrm{O}\right): \delta 58.33\left(\mathrm{CH}_{2} \mathrm{O}\right), 60.55\left(\mathrm{C}_{6^{\prime}}\right), 69.09\left(\mathrm{C}_{4}{ }^{\prime}\right), 72.44\left(\mathrm{C}_{2}\right), 76.04$ $\left(\mathrm{C}_{3},\right), 79.04\left(\mathrm{C}_{5}\right), 87.66\left(\mathrm{C}_{1}{ }^{\prime}\right), 125.21($ triazole $\mathrm{CH}), 126.24(\mathrm{Ph} \mathrm{CH}), 130.62(\mathrm{Ph} \mathrm{CH}), 133.21$ (Ph C), 142.80 (triazole $\mathrm{C}$ or $\mathrm{Ph} \mathrm{C}), 145.67$ (triazole $\mathrm{Ph} \mathrm{C}), 166.64(\mathrm{C}=\mathrm{O})$. HRMS (ESI) calcd for $\mathrm{C}_{16} \mathrm{H}_{19} \mathrm{~N}_{4} \mathrm{O}_{9} \mathrm{~S}^{-}: 443.087822$. Found: 443.087455.

\section{4-(\{[4-(Aminosulfonyl)benzoyl]oxy\}methyl-1-(2'-3'-4'-6'-tetra-O-acetyl-}

galactopyranosyl)-1-H-1,2,3-triazole (3b). The title compound was prepared according to General Procedure 1 and isolated as white foam (184 mg, $0.30 \mathrm{mmol}, 92 \%) . R_{f} 0.41(8: 2$ EtOAc:Hexanes). ${ }^{1} \mathrm{H}$ NMR (400 MHz, DMSO-d $\left.{ }_{6}\right): \delta 1.78$ (s, 3H, OAc $\mathrm{CH}_{3}$ ), 1.92 (s, 3H, $\left.\mathrm{OAc} \mathrm{CH}_{3}\right), 1.96\left(\mathrm{~s}, \mathrm{OAc} \mathrm{CH}_{3}\right), 2.16\left(\mathrm{~s}, \mathrm{OAc} \mathrm{CH}_{3}\right), 4.00\left(\mathrm{dd},{ }^{2} J_{6^{\prime}-6^{\prime}},=11.6 \mathrm{~Hz},{ }^{3} J_{6^{\prime}-5},=1.2 \mathrm{~Hz}\right.$, $\left.1 \mathrm{H}, \mathrm{H}_{6}{ }^{\prime}\right), 4.11\left(\mathrm{dd},{ }^{2} J_{6}{ }^{\prime}-6^{\prime}=11.6 \mathrm{~Hz},{ }^{3} \mathrm{~J}_{6}{ }^{\prime}-5,=4.8 \mathrm{~Hz}, 1 \mathrm{H}, \mathrm{H}_{6}{ }^{\prime}\right), 4.55-4.58\left(\mathrm{~m}, 1 \mathrm{H}, \mathrm{H}_{5}{ }^{\prime}\right), 5.40$ 
$\left(\mathrm{dd},{ }^{3} J_{4^{\prime}-3^{\prime}}=3.6 \mathrm{~Hz},{ }^{3} J_{4^{\prime}-5}{ }^{\prime}=1.2 \mathrm{~Hz}, \mathrm{H}_{4}{ }^{\prime}\right), 5.42\left(\mathrm{~s}, 2 \mathrm{H}, \mathrm{CH}_{2} \mathrm{O}\right), 5.44-5.45\left(\mathrm{~m}, 1 \mathrm{H}, \mathrm{H}_{3}{ }^{\prime}\right), 5.56-$ $5.61\left(\mathrm{~m}, 1 \mathrm{H}, \mathrm{H}_{2^{\prime}}\right), 6.27\left(\mathrm{~d},{ }^{3} J_{1^{\prime}-2^{\prime}}=9.2 \mathrm{~Hz}, 1 \mathrm{H}, \mathrm{H}_{1^{\prime}}\right), 7.53\left(\mathrm{~s}, 2 \mathrm{H}, \mathrm{SO}_{2} \mathrm{NH}_{2}\right), 7.91-8.11(\mathrm{~m}$, $4 \mathrm{H}, \mathrm{Ph} \mathrm{H}), 8.50(\mathrm{~s}, 1 \mathrm{H}$, triazole $\mathrm{H}) ;{ }^{13} \mathrm{C}\left\{{ }^{1} \mathrm{H}\right\}$ NMR $\left(100 \mathrm{MHz}, \mathrm{DMSO}-d_{6}\right): \delta 20.65(\mathrm{OAc}$ $\left.\mathrm{CH}_{3}\right), 20.99\left(\mathrm{OAc} \mathrm{CH}_{3}\right), 21.09\left(\mathrm{OAc} \mathrm{CH}_{3}\right), 21.17\left(\mathrm{OAc} \mathrm{CH}_{3}\right), 58.78\left(\mathrm{CH}_{2} \mathrm{O}\right), 62.25\left(\mathrm{C}_{6}{ }^{\prime}\right)$, $67.98\left(\mathrm{C}_{4}{ }^{\prime}\right), 68.43\left(\mathrm{C}_{3^{\prime}}\right), 71.04\left(\mathrm{C}_{2}\right), 73.70\left(\mathrm{C}_{5}\right), 84.96\left(\mathrm{C}_{1}{ }^{\prime}\right), 125.23$ (triazole $\left.\mathrm{CH}\right), 126.77(\mathrm{Ph}$ $\mathrm{CH}), 130.68(\mathrm{Ph} \mathrm{CH}), 132.70(\mathrm{Ph} \mathrm{C}), 142.92(\mathrm{Ph} \mathrm{C}), 148.91$ (triazole $\mathrm{C}), 165.13(\mathrm{C}=\mathrm{O})$, $169.20(\mathrm{C}=\mathrm{O}), 170.13(\mathrm{C}=\mathrm{O}), 170.60(\mathrm{C}=\mathrm{O}), 170.66(\mathrm{C}=\mathrm{O})$. HRMS (ESI) calculated for $\mathrm{C}_{24} \mathrm{H}_{27} \mathrm{~N}_{4} \mathrm{O}_{13} \mathrm{~S}^{-}:$611.130081. Found: 611.129142. Anal $\left(\mathrm{C}_{24} \mathrm{H}_{28} \mathrm{~N}_{4} \mathrm{O}_{13} \mathrm{~S} \bullet 0.5 \mathrm{H}_{2} \mathrm{O}\right) \mathrm{C}, \mathrm{H}, \mathrm{N}, \mathrm{S}$

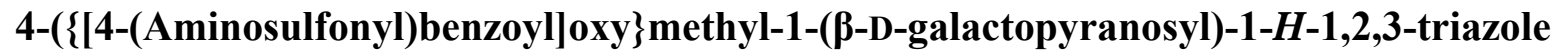

(3b'). The title compound was prepared according to General Procedure 1 and isolated as white foam (136 mg, $0.31 \mathrm{mmol}, 63 \%) . R_{f} 0.12\left(3: 7 \mathrm{EtOH}: \mathrm{CHCl}_{3}\right) .{ }^{1} \mathrm{H} \mathrm{NMR}(400 \mathrm{MHz}, 2 \%$ $\mathrm{D}_{2} \mathrm{O}$ in DMSO- $\left.d_{6}\right): \delta 3.41-3.48\left(\mathrm{~m}, 3 \mathrm{H}, \mathrm{H}_{5}, \mathrm{H}_{6}, \mathrm{H}_{6},{ }^{\prime}\right), 3.51\left(\mathrm{dd},{ }^{3} J_{3^{\prime}-2^{\prime}}=9.2 \mathrm{~Hz},{ }^{3} J_{3^{\prime}-4^{\prime}}=3.2\right.$ $\left.\mathrm{Hz}, 1 \mathrm{H}, \mathrm{H}_{3}{ }^{\prime}\right), 3.67-3.73\left(\mathrm{~m}, 1 \mathrm{H}, \mathrm{H}_{4}{ }^{\prime}\right), 3.98-4.03\left(\mathrm{~m}, 1 \mathrm{H}, \mathrm{H}_{2}\right), 5.43\left(\mathrm{~s}, 2 \mathrm{H}, \mathrm{CH}_{2} \mathrm{O}\right), 5.48(\mathrm{~d}$, $\left.{ }^{3} J_{1^{\prime}-2^{\prime}}=8.8 \mathrm{~Hz}, 1 \mathrm{H}, \mathrm{H}_{1^{\prime}}\right), 7.91-8.12(\mathrm{~m}, 4 \mathrm{H}, \mathrm{Ph}), 8.37(\mathrm{~s}, 1 \mathrm{H}$, triazole $\mathrm{CH}) ;{ }^{13} \mathrm{C}\left\{{ }^{1} \mathrm{H}\right\} \mathrm{NMR}$ $\left.\left(100 \mathrm{MHz}, 2 \% \mathrm{D}_{2} \mathrm{O} \text { in DMSO-d }\right)_{6}\right): \delta 58.42\left(\mathrm{CH}_{2} \mathrm{O}\right), 60.38\left(\mathrm{C}_{6}{ }^{\prime}\right), 68.39\left(\mathrm{C}_{4}{ }^{\prime}\right), 69.24\left(\mathrm{C}_{2}{ }^{\prime}\right)$, $73.52\left(\mathrm{C}_{3}\right), 78.46\left(\mathrm{C}_{5}{ }^{\prime}\right), 88.19\left(\mathrm{C}_{1}{ }^{\prime}\right), 124.01($ triazole $\mathrm{CH}), 126.21(\mathrm{Ph} \mathrm{CH}), 130.10(\mathrm{Ph} \mathrm{CH})$, 132.16 ( $\mathrm{Ph} \mathrm{C}$ ), 141.69 ( $\mathrm{Ph} \mathrm{C}), 148.13$ (triazole $\mathrm{C}), 164.62(\mathrm{C}=\mathrm{O})$. HRMS (ESI) calcd for $\mathrm{C}_{16} \mathrm{H}_{19} \mathrm{~N}_{4} \mathrm{O}_{9} \mathrm{~S}^{-}: 443.087822$. Found: 443.087859.

4-(\{[4-(Aminosulfonyl)benzoyl]oxy\}methyl-1-(2'-3'-4'tri-O-acetylarabinopyranosyl)-1-H-1,2,3-triazole (3c). The title compound was prepared according to General Procedure 1 and isolated as white foam (228 mg, $0.42 \mathrm{mmol}, 88 \%) . R_{f} 0.55(8: 2$ EtOAc:Hexanes). ${ }^{1} \mathrm{H}$ NMR (400 MHz, DMSO-d $\left.)_{6}\right): 1.78$ (s, 3H, OAc $\mathrm{CH}_{3}$ ), 1.93 (s, 3H, OAc $\left.\mathrm{CH}_{3}\right), 2.13\left(\mathrm{~s}, 3 \mathrm{H}, \mathrm{OAc} \mathrm{CH}_{3}\right), 4.02\left(\mathrm{dd},{ }^{2} J_{5^{\prime}-5}{ }^{\prime}=13.2 \mathrm{~Hz},{ }^{3} J_{5}{ }^{\prime}-4,=1.6 \mathrm{~Hz}, 1 \mathrm{H}, \mathrm{H}_{5}{ }^{\prime}\right), 4.14-4.18$ 
$\left(\mathrm{m}, 1 \mathrm{H}, \mathrm{H}_{5^{\prime}}\right), 5.28-5.30\left(\mathrm{~m}, 1 \mathrm{H}, \mathrm{H}_{4^{\prime}}\right), 5.38\left(\mathrm{dd},{ }^{3} J_{3^{\prime}-2^{\prime}}=10.0 \mathrm{~Hz},{ }^{3} J_{3^{\prime}-4}{ }^{\prime}=3.6 \mathrm{~Hz}, 1 \mathrm{H}, \mathrm{H}_{3^{\prime}}\right)$, $5.42\left(\mathrm{~s}, 2 \mathrm{H}, \mathrm{CH}_{2} \mathrm{O}\right), 5.55-5.60\left(\mathrm{~m}, 1 \mathrm{H}, \mathrm{H}_{2}\right), 6.13\left(\mathrm{~d},{ }^{3} \mathrm{~J}_{1^{\prime}-2^{\prime}}=9.2 \mathrm{~Hz}, 1 \mathrm{H}, \mathrm{H}_{1}{ }^{\prime}\right), 7.53($ br s, $2 \mathrm{H}$, $\left.\mathrm{SO}_{2} \mathrm{NH}_{2}\right), 7.91-8.10(\mathrm{~m}, 4 \mathrm{H}, \mathrm{Ph} \mathrm{CH}), 8.46(\mathrm{~s}, 1 \mathrm{H}$, triazole $\mathrm{H}) ;{ }^{13} \mathrm{C}\left\{{ }^{1} \mathrm{H}\right\} \quad \mathrm{NMR}(100 \mathrm{MHz}$, DMSO-d $\left.{ }_{6}\right): \delta 20.66\left(\mathrm{OAc} \mathrm{CH}_{3}\right), 21.07\left(\mathrm{OAc} \mathrm{CH}_{3}\right), 21.40\left(\mathrm{OAc} \mathrm{CH}_{3}\right), 58.81\left(\mathrm{CH}_{2} \mathrm{O}\right), 67.18$ $\left(\mathrm{C}_{5^{\prime}}\right), 68.57\left(\mathrm{C}_{4^{\prime}}\right), 68.69\left(\mathrm{C}_{2}\right), 70.95\left(\mathrm{C}_{3^{\prime}}\right), 85.52\left(\mathrm{C}_{1^{\prime}}\right), 125.11($ triazole $\mathrm{CH}), 126.76(\mathrm{Ph} \mathrm{CH})$, $130.67(\mathrm{Ph} \mathrm{CH}), 132.71(\mathrm{Ph} \mathrm{C}), 142.86(\mathrm{Ph} \mathrm{C}), 148.91$ (triazole $\mathrm{CH}), 165.11(\mathrm{C}=\mathrm{O}), 169.24$ $(\mathrm{C}=\mathrm{O}), \quad 170.20(\mathrm{C}=\mathrm{O}), \quad 170.55(\mathrm{C}=\mathrm{O})$. HRMS (ESI) calculated for $\mathrm{C}_{21} \mathrm{H}_{23} \mathrm{~N}_{4} \mathrm{O}_{11} \mathrm{~S}^{-}$: 539.108952. Found: 539.107401. Anal $\left(\mathrm{C}_{21} \mathrm{H}_{24} \mathrm{~N}_{4} \mathrm{O}_{11} \mathrm{~S} \bullet 1.5 \mathrm{H}_{2} \mathrm{O}\right) \mathrm{C}, \mathrm{H}, \mathrm{N}, \mathrm{S}$.

\section{4-(\{[4-(Aminosulfonyl)benzoyl $]$ oxy $\}$ methyl-1-( $\beta$-D-arabinopyranosyl)-1-H-1,2,3-triazole}

(3c'). The title compound was prepared according to procedure 1 and isolated as white solid (105 mg, $0.25 \mathrm{mmol}, 84 \%) . R_{f} 0.26$ (3:7 EtOH:CHCl$)$; mp $184-189{ }^{\circ} \mathrm{C} .{ }^{1} \mathrm{H}$ NMR (400 $\mathrm{MHz}, 2 \% \mathrm{D}_{2} \mathrm{O}$ in DMSO- $\left.d_{6}\right): \delta 3.53\left(\mathrm{dd},{ }^{3} J_{3^{\prime}-{ }^{\prime}}{ }^{\prime}=9.6 \mathrm{~Hz},{ }^{3} J_{3^{\prime}-4^{\prime}}=3.6 \mathrm{~Hz}, 1 \mathrm{H}, \mathrm{H}_{3^{\prime}}\right), 3.72-3.74$ $\left(\mathrm{m}, 2 \mathrm{H}, \mathrm{H}_{4}, \mathrm{H}_{5}\right.$ ) $, 3.78\left(\mathrm{dd},{ }^{2} J_{5}{ }^{\prime}-5^{\prime}=12.4 \mathrm{~Hz},{ }^{3} J_{5^{\prime}-4^{\prime}}=2.0 \mathrm{~Hz}, 1 \mathrm{H}, \mathrm{H}_{5},\right), 3.98-4.03(\mathrm{~m}, 1 \mathrm{H}$, $\left.\mathrm{H}_{2}{ }^{\prime}\right), 5.41\left(\mathrm{~d},{ }^{3} J_{1^{\prime}-2^{\prime}}=10.0 \mathrm{~Hz}, 1 \mathrm{H}, \mathrm{H}_{1}{ }^{\prime}\right), 5.43\left(\mathrm{~s}, 2 \mathrm{H}, \mathrm{CH}_{2} \mathrm{O}\right), 8.36(\mathrm{~s}, 1 \mathrm{H}$, triazole $\mathrm{CH}), 7.91-$ $8.12(\mathrm{~m}, 4 \mathrm{H}, \mathrm{Ph}) ;{ }^{13} \mathrm{C}\left\{{ }^{1} \mathrm{H}\right\}$ NMR (100 MHz, $2 \% \mathrm{D}_{2} \mathrm{O}$ in DMSO-d 6$): \delta 59.06\left(\mathrm{CH}_{2} \mathrm{O}\right), 69.03$ $\left(\mathrm{C}_{4},\right), 69.96\left(\mathrm{C}_{5}, \mathrm{C}_{2},\right), 73.86\left(\mathrm{C}_{3}{ }^{\prime}\right), 89.10\left(\mathrm{C}_{1}{ }^{\prime}\right), 124.59($ triazole $\mathrm{CH}), 126.79(\mathrm{Ph} \mathrm{CH}), 130.70$ (Ph CH), $132.74(\mathrm{Ph} \mathrm{C}), 142.25$ (triazole $\mathrm{C}$ or $\mathrm{Ph} \mathrm{C}$ ), 148.81 (triazole $\mathrm{C}$ or $\mathrm{Ph} \mathrm{C}), 165.21$ $(\mathrm{C}=\mathrm{O})$. HRMS (ESI) calcd for $\mathrm{C}_{15} \mathrm{H}_{17} \mathrm{~N}_{4} \mathrm{O}_{8} \mathrm{~S}^{-}:$413.077258. Found: 413.076552.

\section{4-(\{[4-(Aminosulfonyl)benzoyl]oxy\}methyl-1-(2'-acetamido-2'-deoxy-3',4',6'-tri-O-}

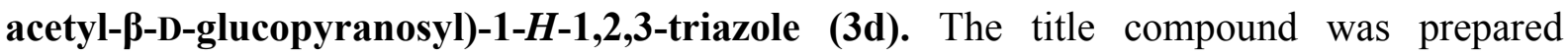
according to the General Procedure 1 and isolated as white solid (292 mg, $0.60 \mathrm{mmol}, 89 \%$ ). $R_{f} 0.45$ (100\% EtOAc); mp 217 - $218{ }^{\circ} \mathrm{C} .{ }^{1} \mathrm{H}$ NMR (400 MHz, DMSO-d 6 ): $\delta 1.55$ (s, 3H, NHAc $\mathrm{CH}_{3}$ ), 1.92 (s, 3H, OAc $\mathrm{CH}_{3}$ ), 1.97 (s, 3H, OAc $\mathrm{CH}_{3}$ ), 1.98 (s, $\mathrm{OAc}_{3} \mathrm{CH}_{3}$ ), 4.03 (dd, 
$\left.{ }^{2} J_{6^{\prime}-6^{\prime}}=12.4 \mathrm{~Hz},{ }^{3} J_{6^{\prime}-5^{\prime}}=2 \mathrm{~Hz}, 1 \mathrm{H}, \mathrm{H}_{6^{\prime}}\right), 4.12\left(\mathrm{dd},{ }^{2} J_{6^{\prime}-5},=12.8 \mathrm{~Hz},{ }^{3} J_{6^{\prime}}{ }^{-5},=5.6 \mathrm{~Hz}, 1 \mathrm{H}, \mathrm{H}_{6^{\prime}}\right)$, $4.23\left(\mathrm{ddd},{ }^{3} J_{5^{\prime}-4},=10.0 \mathrm{~Hz},{ }^{3} J_{5^{\prime}-6^{\prime}},=5.6 \mathrm{~Hz},{ }^{3} J_{5^{\prime}-6^{\prime}}=2.0 \mathrm{~Hz}, 1 \mathrm{H}, \mathrm{H}_{5^{\prime}}\right), 4.56-4.64(\mathrm{~m}, 1 \mathrm{H}$, $\left.\mathrm{H}_{2}\right), 5.06-5.10\left(\mathrm{~m}, 1 \mathrm{H}, \mathrm{H}_{4}\right), 5.30-5.35\left(\mathrm{~m}, 1 \mathrm{H}, \mathrm{H}_{3}{ }^{\prime}\right), 5.38-5.45\left(\mathrm{~m}, 2 \mathrm{H}, \mathrm{CH}_{2} \mathrm{O}\right), 6.11(\mathrm{~d}$, ${ }^{3} J_{1^{\prime}-2},=9.6 \mathrm{~Hz}, 1 \mathrm{H}, \mathrm{H}_{1}$ ) $), 7.54\left(\mathrm{~s}, 2 \mathrm{H}, \mathrm{SO}_{2} \mathrm{NH}_{2}\right), 7.91-8.12(\mathrm{~m}, 4 \mathrm{H}, \mathrm{Ph} \mathrm{H}), 8.05\left(\mathrm{~d},{ }^{3} J_{\mathrm{NH}-2}=\right.$ $9.6 \mathrm{~Hz}, 1 \mathrm{H}, \mathrm{NHAc} \mathrm{CONH}), 8.44(\mathrm{~s}$, triazole $\mathrm{H}) ;{ }^{13} \mathrm{C}\left\{{ }^{1} \mathrm{H}\right\}$ NMR $\left(100 \mathrm{MHz}, \mathrm{DMSO}-d_{6}\right): \delta$ $20.96\left(\mathrm{OAc}_{3}\right), 21.10\left(\mathrm{OAc}_{3} \mathrm{CH}_{3}\right), 21.20\left(\mathrm{OAc}_{\mathrm{CH}}\right), 22.97\left(\mathrm{NHAc} \mathrm{CH}_{3}\right), 52.74\left(\mathrm{C}_{2}{ }^{\prime}\right)$, $58.96\left(\mathrm{CH}_{2} \mathrm{O}\right), 62.45\left(\mathrm{C}_{6^{\prime}}\right), 68.66\left(\mathrm{C}_{3^{\prime}}\right), 72.99\left(\mathrm{C}_{4^{\prime}}\right), 74.10\left(\mathrm{C}_{5}\right), 85.45\left(\mathrm{C}_{1}{ }^{\prime}\right), 124.49$ (triazole $\mathrm{CH}), 126.75(\mathrm{Ph} \mathrm{CH}), 130.70(\mathrm{Ph} \mathrm{CH}), 132.71(\mathrm{Ph} \mathrm{CH}), 142.64$ (Ph C), 148.91 (triazole $\mathrm{C})$, $165.10(\mathrm{C}=\mathrm{O}), 170.02(\mathrm{C}=\mathrm{O}), 170.10(\mathrm{C}=\mathrm{O}), 170.27(\mathrm{C}=\mathrm{O}), 170.71(\mathrm{C}=\mathrm{O})$. HRMS (ESI) calcd for $\mathrm{C}_{24} \mathrm{H}_{28} \mathrm{~N}_{5} \mathrm{O}_{12} \mathrm{~S}^{-}:$610.146066. Found: 610.145354. Anal $\left(\mathrm{C}_{24} \mathrm{H}_{29} \mathrm{~N}_{5} \mathrm{O}_{12} \mathrm{~S} \bullet 0.5 \mathrm{H}_{2} \mathrm{O}\right) \mathrm{C}$, H, N, S.

\section{4-(\{[4-(Aminosulfonyl)benzoyl]oxy\}methyl-1-(2'-acetamido-2'-deoxy- $\beta$-D-}

glucopyranosyl)-1-H-1,2,3-triazole (3d'). The title compound was prepared according to the General Procedure 1 and isolated as off-white solid (180 mg, $0.38 \mathrm{mmol}, 68 \%) R_{f} 0.09$ (3:7 EtOH:CHCl 3 ); mp $205-208{ }^{\circ} \mathrm{C}$ (decomp). ${ }^{1} \mathrm{H}$ NMR (400 MHz, 2\% $\mathrm{D}_{2} \mathrm{O}$ in DMSO- $d_{6}$,): $\delta$ $1.57\left(\mathrm{~s}, 3 \mathrm{H}, \mathrm{NHAc} \mathrm{CH}_{3}\right), 3.22-3.27\left(\mathrm{~m}, 1 \mathrm{H}, \mathrm{H}_{4}{ }^{\prime}\right), 3.68-3.46\left(\mathrm{~m}, 2 \mathrm{H}, \mathrm{H}_{5}, \mathrm{H}_{6}{ }^{\prime}\right), 3.49-3.54$ (m, $\left.1 \mathrm{H}, \mathrm{H}_{3^{\prime}}\right), 3.65-3.69\left(\mathrm{~m}, 1 \mathrm{H}, \mathrm{H}_{6^{\prime}}\right.$ ) $), 4.01-4.08\left(\mathrm{~m}, 1 \mathrm{H}, \mathrm{H}_{2^{\prime}}\right), 5.39\left(\mathrm{~s}, 2 \mathrm{H}, \mathrm{CH}_{2} \mathrm{O}\right), 5.71(\mathrm{~d}$, $\left.{ }^{3} J_{1^{\prime}-2},=9.6 \mathrm{~Hz}, 1 \mathrm{H}, \mathrm{H}_{1}{ }^{\prime}\right), 7.85\left(\mathrm{~d},{ }^{3} J_{\mathrm{NH}-2}=9.2 \mathrm{~Hz}, 1 \mathrm{H}, \mathrm{NHAc} \mathrm{CONH}\right), 7.91-8.10(\mathrm{~m}, 4 \mathrm{H}$, $\mathrm{Ph}), 8.27(\mathrm{~s}, 1 \mathrm{H}$, triazole $\mathrm{CH}) ;{ }^{13} \mathrm{C}\left\{{ }^{1} \mathrm{H}\right\}$ NMR $\left(100 \mathrm{MHz}, \mathrm{DMSO}-d_{6}\right): \delta 23.36\left(\mathrm{NHAc}_{\mathrm{CH}}\right)$, $55.20\left(\mathrm{C}_{2}{ }^{\prime}\right), 58.99\left(\mathrm{CH}_{2} \mathrm{O}\right), 61.35\left(\mathrm{C}_{6^{\prime}}\right), 70.61\left(\mathrm{C}_{4}{ }^{\prime}\right), 74.57\left(\mathrm{C}_{3^{\prime}}\right), 80.81\left(\mathrm{C}_{5^{\prime}}\right), 86.74\left(\mathrm{C}_{1^{\prime}}\right)$, 124.34 (triazole $\mathrm{CH}), 126.76(\mathrm{Ph} \mathrm{CH}), 130.69(\mathrm{Ph} \mathrm{CH}), 132.76(\mathrm{Ph} \mathrm{C}), 142.10$ (triazole $\mathrm{C}$ or Ph C), 148.87 (triazole C), $165.13(\mathrm{C}=\mathrm{O}), 169.82(\mathrm{C}=\mathrm{O}) . \quad$ HRMS (ESI) calcd for $\mathrm{C}_{18} \mathrm{H}_{22} \mathrm{~N}_{5} \mathrm{O}_{9} \mathrm{~S}^{-}:$484.114372. Found: 484.113226. 
4-(\{[4-(Aminosulfonyl)benzoyl $]$ oxy $\}$ methyl-1-(2',3',4'-tri-O-acetyl- $\beta$-D-glucuronic acid methyl ester)-1-H-1,2,3-triazole (3e). The title compound was prepared according to General Procedure 1 and isolated as white foam $(307 \mathrm{mg}, 92 \%) . R_{f} 0.58\left(2: 8\right.$ Hexanes:EtOAc). ${ }^{1} \mathrm{H}$ NMR (400 MHz, DMSO-d $\left.)_{6}\right): \delta 1.76\left(\mathrm{~s}, 3 \mathrm{H}, \mathrm{OAc} \mathrm{CH}_{3}\right), 1.96\left(\mathrm{~s}, 3 \mathrm{H}, \mathrm{OAc} \mathrm{CH}_{3}\right), 1.99(\mathrm{~s}, 3 \mathrm{H}$, $\left.\mathrm{OAc} \mathrm{CH}_{3}\right), 3.60\left(\mathrm{~s}, 3 \mathrm{H}, \mathrm{COCH}_{3}\right), 4.79\left(\mathrm{~d},{ }^{3} \mathrm{~J}_{5^{\prime}-4}{ }^{\prime}=10.0 \mathrm{~Hz}, 1 \mathrm{H}, \mathrm{H}_{5}{ }^{\prime}\right), 5.21-5.26\left(\mathrm{~m}, 1 \mathrm{H}, \mathrm{H}_{4}{ }^{\prime}\right)$, $5.43\left(\mathrm{~s}, 2 \mathrm{H}, \mathrm{CH}_{2} \mathrm{O}\right), 5.57-5.62\left(\mathrm{~m}, 1 \mathrm{H}, \mathrm{H}_{3^{\prime}}\right), 5.73-5.78\left(\mathrm{~m}, 1 \mathrm{H}, \mathrm{H}_{2}{ }^{\prime}\right), 6.40\left(\mathrm{~d}^{3}{ }^{3} J_{1^{\prime}-2^{\prime}}=9.2\right.$ $\mathrm{Hz}, 1 \mathrm{H}, \mathrm{H}_{1}$ ) $) 7.54$ (br s, 2H, $\left.\mathrm{SO}_{2} \mathrm{NH}_{2}\right), 7.91-8.12$ (m, 4H, Ph H), 8.65 (s, 1H, triazole $\left.\mathrm{H}\right)$;

${ }^{13} \mathrm{C}\left\{{ }^{1} \mathrm{H}\right\}$ NMR (100 MHz, DMSO-d 6$): \delta 20.56\left(\mathrm{OAc} \mathrm{CH}_{3}\right), 20.90\left(\mathrm{OAc} \mathrm{CH}_{3}\right), 20.95(\mathrm{OAc}$ $\left.\mathrm{CH}_{3}\right), 53.30\left(\mathrm{COCH}_{3}\right), 58.90\left(\mathrm{CH}_{2} \mathrm{O}\right), 69.05\left(\mathrm{C}_{4}{ }^{\prime}\right), 70.48\left(\mathrm{C}_{3}{ }^{\prime}\right), 72.13\left(\mathrm{C}_{2}\right), 73.56\left(\mathrm{C}_{5}\right), 84.48$ $\left(\mathrm{C}_{1}{ }^{\circ}\right), 124.90$ (triazole $\left.\mathrm{CH}\right), 126.77(\mathrm{Ph} \mathrm{CH}), 130.69(\mathrm{Ph} \mathrm{CH}), 132.67(\mathrm{Ph} \mathrm{C}), 143.28$ (triazole $\mathrm{C}$ or $\mathrm{Ph} \mathrm{C}), 148.92$ (triazole $\mathrm{C}$ or $\mathrm{Ph} \mathrm{C}), 165.09(\mathrm{C}=\mathrm{O}), 167.24(\mathrm{C}=\mathrm{O}), 169.10(\mathrm{C}=\mathrm{O}), 169.98$ $(\mathrm{C}=\mathrm{O}), 170.19(\mathrm{C}=\mathrm{O})$. HRMS (ESI) calculated for $\mathrm{C}_{23} \mathrm{H}_{25} \mathrm{~N}_{4} \mathrm{O}_{13} \mathrm{SCl}^{-}$: 633.091109. Found: .633.094227. Anal $\left(\mathrm{C}_{23} \mathrm{H}_{26} \mathrm{~N}_{4} \mathrm{O}_{13} \mathrm{~S} \bullet 1.5 \mathrm{H}_{2} \mathrm{O}\right) \mathrm{C}, \mathrm{H}, \mathrm{N}, \mathrm{S}$.

\section{4-(\{[4-(Aminosulfonyl)benzoyl]oxy\}methyl-1-( $\beta$-D-glucuronic acid methyl ester)-1-H-}

1,2,3-triazole (3e'). The title compound was prepared according to General Procedure 1 and isolated as hygroscopic white foam (42 mg, $0.08 \mathrm{mmol}, 72 \%) . R_{f} 0.32$ (1:9 MeOH:EtOAc). ${ }^{1} \mathrm{H}$ NMR $\left(400 \mathrm{MHz}, 2 \% \mathrm{D}_{2} \mathrm{O}\right.$ in DMSO-d $\left.\mathrm{D}_{6}\right): \delta 3.27-3.32\left(\mathrm{~m}, 1 \mathrm{H}, \mathrm{H}_{3}\right), 3.96-3.01(\mathrm{~m}, 1 \mathrm{H}$, $\left.\mathrm{H}_{4}{ }^{\prime}\right), 3.65\left(\mathrm{~s}, 3 \mathrm{H}, \mathrm{COCH}_{3}\right), 3.84-3.88\left(\mathrm{~m}, 1 \mathrm{H}, \mathrm{H}_{2^{\prime}}\right), 4.63\left(\mathrm{~d},{ }^{3} J_{5^{\prime}-4^{\prime}}=9.6 \mathrm{~Hz}, 1 \mathrm{H}, \mathrm{H}_{5^{\prime}}\right), 5.42(\mathrm{~s}$, $\left.2 \mathrm{H}, \mathrm{CH}_{2} \mathrm{O}\right), 5.72\left(\mathrm{~d},{ }^{3} \mathrm{~J}_{1^{\prime}-2^{\prime}}=9.6 \mathrm{~Hz}, 1 \mathrm{H}, \mathrm{H}_{1^{\prime}}\right), 7.91-8.12(\mathrm{~m}, 4 \mathrm{H}, \mathrm{Ph} \mathrm{H}), 8.46(\mathrm{~s}, 1 \mathrm{H}$, triazole $\mathrm{CH}) ;{ }^{13} \mathrm{C}\left\{{ }^{1} \mathrm{H}\right\}$ NMR $\left(100 \mathrm{MHz}, 2 \% \mathrm{D}_{2} \mathrm{O}\right.$ in DMSO-d $\left.d_{6}\right): \delta 52.81\left(\mathrm{COCH}_{3}\right), 58.92\left(\mathrm{CH}_{2} \mathrm{O}\right)$, $71.56\left(\mathrm{C}_{3}\right), 71.89\left(\mathrm{C}_{4}{ }^{\prime}\right), 76.68\left(\mathrm{C}_{2}\right), 78.00\left(\mathrm{C}_{5},\right), 87.79\left(\mathrm{C}_{1},\right), 125.09$ (triazole $\left.\mathrm{CH}\right), 126.80(\mathrm{Ph}$ $\mathrm{CH}), 130.73(\mathrm{Ph} \mathrm{CH}), 136.85(\mathrm{Ph} \mathrm{C}), 142.40$ (triazole $\mathrm{C}$ or $\mathrm{Ph} \mathrm{C}), 148.49$ (triazole $\mathrm{C}$ or $\mathrm{Ph}$ C), $165.20(\mathrm{C}=\mathrm{O}), 169.35(\mathrm{C}=\mathrm{O})$. HRMS (ESI) calcd for $\mathrm{C}_{17} \mathrm{H}_{19} \mathrm{~N}_{4} \mathrm{O}_{10} \mathrm{~S}^{-}$: 471.082737 . Found: 471.082075 . 


\section{4-(\{[4-(Aminosulfonyl)benzoyl]oxy\} methyl-1-(hepta-O-acetyl- $\beta-D-m a l t o s y l)-1-H-1,2,3-$}

triazole (3f). The title compound was prepared according to General Procedure 1 and isolated as white foam (185 mg, $0.21 \mathrm{mmol}, 91 \%) . R_{f} 0.46$ (8:2 EtOAc:Hexanes). ${ }^{1} \mathrm{H}$ NMR (400 MHz, DMSO-d $\left.d_{6}\right): \delta 1.71\left(\mathrm{~s}, 3 \mathrm{H}, \mathrm{OAc}_{\mathrm{CH}}\right), 1.93\left(\mathrm{~s}, 6 \mathrm{H}, 2\right.$ x $\left.\mathrm{OAc}_{\mathrm{CH}}\right), 1.96(\mathrm{~s}, 3 \mathrm{H}, \mathrm{OAc}$ $\left.\mathrm{CH}_{3}\right), 1.97$ (s, 3H, OAc $\left.\mathrm{CH}_{3}\right), 1.99\left(\mathrm{~s}, 3 \mathrm{H}, \mathrm{OAc} \mathrm{CH}_{3}\right), 2.02\left(\mathrm{~s}, 3 \mathrm{H}, \mathrm{OAc} \mathrm{CH}_{3}\right), 3.97-4.02(\mathrm{~m}$, 2H, Glc $\alpha \mathrm{H}_{5}$, Glc $\beta \mathrm{H}_{6}{ }^{\prime}$ ), 4.07 - 4.17 (m, 3H, Glc $\alpha \mathrm{H}_{6^{\prime}}$, Glc $\beta \mathrm{H}_{4}$, , Glc $\beta \mathrm{H}_{6}{ }^{\prime}$ ), $4.32\left(\mathrm{ddd},{ }^{3} J_{5}{ }^{\prime}-4^{\prime},=\right.$ $\left.10 \mathrm{~Hz},{ }^{3} J_{5^{\prime}-6^{\prime}}=5.6 \mathrm{~Hz},{ }^{3} J_{5^{\prime}-6^{\prime}},=2.4 \mathrm{~Hz}, 1 \mathrm{H}, \mathrm{Glc} \beta \mathrm{H}_{5},\right), 4.42\left(\mathrm{dd},{ }^{2} J_{6^{\prime}}{ }^{\prime} 6^{\prime}=12.4 \mathrm{~Hz},{ }^{3} J_{6^{\prime}-}-^{\prime},=2\right.$ $\mathrm{Hz}, 1 \mathrm{H}, \mathrm{Glc} \beta \mathrm{H}_{6^{\prime}}$ ), 4.89 (dd, $1 \mathrm{H},{ }^{3} J_{2^{\prime}-3^{\prime}}=10.4 \mathrm{~Hz},{ }^{3} J_{2^{\prime}-1}$, $=3.6 \mathrm{~Hz}, 1 \mathrm{H}, \mathrm{Glc} \alpha \mathrm{H}_{2^{\prime}}$ ), $4.96-5.01$ $\left(\mathrm{m}, 1 \mathrm{H}, \mathrm{Glc} \alpha \mathrm{H}_{4^{\prime}}\right), 5.19-5.24\left(\mathrm{~m}, 1 \mathrm{H}, \mathrm{Glc} \alpha \mathrm{H}_{3^{\prime}}\right), 5.33\left(\mathrm{~d},{ }^{3} J_{1^{\prime}-2^{\prime}}=4.0 \mathrm{~Hz}, 1 \mathrm{H}, \mathrm{Glc} \alpha \mathrm{H}_{1^{\prime}}\right)$, $5.38-5.45\left(\mathrm{~m}, 2 \mathrm{H}, \mathrm{CH}_{2} \mathrm{O}\right), 5.49-5.53\left(\mathrm{~m}, 1 \mathrm{H}, \mathrm{Glc} \beta \mathrm{H}_{2}\right), 5.55-5.60\left(\mathrm{~m}, 1 \mathrm{H}, \mathrm{Glc} \beta \mathrm{H}_{3}{ }^{\prime}\right)$, $6.31\left(\mathrm{~d},{ }^{3} J_{1^{\prime}-2^{\prime}}=8.8 \mathrm{~Hz}, 1 \mathrm{H}, \mathrm{Glc} \beta \mathrm{H}_{1^{\prime}}\right), 7.54\left(\mathrm{~s}, 2 \mathrm{H}, \mathrm{SO}_{2} \mathrm{NH}_{2}\right), 7.91-8.10(\mathrm{~m}, 4 \mathrm{H}, \mathrm{Ph} \mathrm{CH})$, $8.47(\mathrm{~s}, 1 \mathrm{H}$, triazole $\mathrm{H}) ;{ }^{13} \mathrm{C}\left\{{ }^{1} \mathrm{H}\right\}$ NMR $\left(100 \mathrm{MHz}, \mathrm{DMSO}-d_{6}\right): \delta 20.57\left(\mathrm{OAc} \mathrm{CH}_{3}\right), 20.95$ $\left(\mathrm{OAc} \mathrm{CH}_{3}\right), 21.01\left(\mathrm{OAc} \mathrm{CH}_{3}\right), 21.02\left(\mathrm{OAc} \mathrm{CH}_{3}\right), 21.12\left(\mathrm{OAc} \mathrm{CH}_{3}\right), 21.19\left(\mathrm{OAc} \mathrm{CH}_{3}\right), 21.25$ $\left(\mathrm{OAc}_{3}\right), 58.91\left(\mathrm{CH}_{2} \mathrm{O}\right), 62.06\left(\mathrm{Glc}_{3} \mathrm{C}_{6}{ }\right), 63.45$ (Glc $\left.\beta \mathrm{C}_{6}{ }^{\prime}\right), 68.37$ (Glc $\left.\alpha \mathrm{C}_{4}{ }^{\prime}\right), 68.85$ (Glc $\beta$

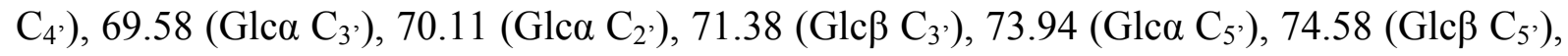
$\left.74.96\left(\mathrm{Glc} \mathrm{C}_{5^{\prime}}\right), 79.85\left(\mathrm{Glc} \beta \mathrm{C}_{2}{ }^{\prime}\right), 84.16(\mathrm{Glc}) \mathrm{C}_{1^{\prime}}\right), 96.41\left(\mathrm{Glc} \mathrm{C}_{1^{\prime}}\right), 124.74$ (triazole $\left.\mathrm{CH}\right)$, 126.76 (Ph CH), 130.67 (Ph CH), 132.68 (Ph C), 143.06 (Ph C), 148.91 (triazole C), 165.09 $(\mathrm{C}=\mathrm{O}), 169.36(\mathrm{OAc} \mathrm{C}=\mathrm{O}), 169.84(\mathrm{OAc} \mathrm{C}=\mathrm{O}), 170.19(\mathrm{OAc} \mathrm{C}=\mathrm{O}), 170.35(\mathrm{C}=\mathrm{O}), 170.53$ $(\mathrm{C}=\mathrm{O}), 170.67(\mathrm{C}=\mathrm{O}), 170.78(\mathrm{C}=\mathrm{O})$. HRMS (ESI) calcd for $\mathrm{C}_{36} \mathrm{H}_{43} \mathrm{~N}_{4} \mathrm{O}_{21} \mathrm{~S}^{-}: 899.214599$. Found: 899.211425. Anal. $\left(\mathrm{C}_{36} \mathrm{H}_{44} \mathrm{~N}_{4} \mathrm{O}_{21} \mathrm{~S} \bullet 1.5 \mathrm{H}_{2} \mathrm{O}\right) \mathrm{C}, \mathrm{H}, \mathrm{N}, \mathrm{S}$.

4-(\{[4-(Aminosulfonyl)benzoyl]oxy\}methyl-1-( $\beta$-D-maltosyl)-1-H-1,2,3-triazole (3f'). The title compound was prepared according to General Procedure 1 and isolated as white foam (161 mg, 0.26 mmol, $58 \%$ \%). $R_{f} 0.39$ (2:8 MeOH:EtOAc). ${ }^{1} \mathrm{H}$ NMR (400 MHz, $\left.\mathrm{D}_{2} \mathrm{O}\right): \delta 3.27-$ 
$3.32\left(\mathrm{~m}, 1 \mathrm{H}, \mathrm{Glc} \alpha \mathrm{H}_{4}\right.$, or Glc $\beta \mathrm{H}_{4}$ ), $3.55-3.79$ (m, 8 H, m, 8H, Glc $\alpha \mathrm{H}_{3}$, Glc $\alpha \mathrm{H}_{5}$, Glc $\alpha$ $\mathrm{H}_{6} / \mathrm{H}_{6}$, , and Glc $\left.\beta \mathrm{H}_{3}{ }^{\prime}, \operatorname{Glc} \beta \mathrm{H}_{5}{ }^{\prime}, \mathrm{Glc} \beta \mathrm{H}_{6}, \mathrm{H}_{6^{\prime}}{ }^{\prime}\right), 3.46\left(\mathrm{dd},{ }^{3} J_{2^{\prime}-3},=10.0 \mathrm{~Hz},{ }^{3} J_{2^{\prime}-1},=3.6 \mathrm{~Hz}, 1 \mathrm{H}\right.$, Glc $\left.\alpha \mathrm{H}_{2}{ }^{\prime}\right), 3.84-3.94\left(\mathrm{~m}, 2 \mathrm{H}, \mathrm{Glc} \beta \mathrm{H}_{2}{ }^{\prime}\right), 3.84-3.94\left(\mathrm{Glc} \beta \mathrm{H}_{2}\right.$, and $\left.\mathrm{Glc} \beta \mathrm{H}_{3^{\prime}}\right), 5.34\left(\mathrm{~d},{ }^{3} J_{1^{\prime}-2^{\prime}}\right.$ $\left.=3.6 \mathrm{~Hz}, 1 \mathrm{H}, \mathrm{Glc} \alpha \mathrm{H}_{1}{ }^{\prime}\right), 5.39\left(\mathrm{~s}, 2 \mathrm{H}, \mathrm{CH}_{2} \mathrm{O}\right), 5.66\left(\mathrm{~d},{ }^{3} J_{1^{\prime}-2}{ }^{\prime}=8.8 \mathrm{~Hz}, 1 \mathrm{H}, \mathrm{Glc} \beta \mathrm{H}_{1^{\prime}}\right), 7.78-$ $7.97(\mathrm{~m}, 4 \mathrm{H}, \mathrm{Ph} \mathrm{H}), 8.25(\mathrm{~s}, 1 \mathrm{H}$, triazole $\mathrm{CH}) ;{ }^{13} \mathrm{C}\left\{{ }^{1} \mathrm{H}\right\} \mathrm{NMR}\left(100 \mathrm{MHz}, \mathrm{D}_{2} \mathrm{O}\right): \delta 58.33$ $\left(\mathrm{CH}_{2} \mathrm{O}\right), 60.54\left(\mathrm{Glc} \mathrm{C}_{6}\right.$, or $\left.\mathrm{Glc} \beta \mathrm{C}_{6}\right), 69.47,71.84,72.31,72.92,72.99,75.93,76.52,77.66$

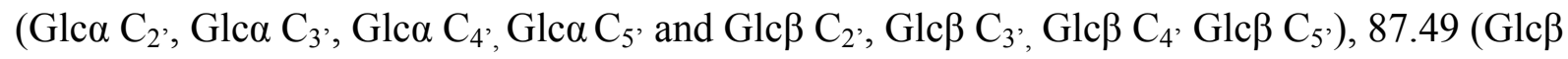
$\mathrm{C}_{1}$ ), $99.81\left(\mathrm{Glc} \alpha \mathrm{C}_{2}\right), 125.16$ (triazole $\left.\mathrm{CH}\right), 126.23(\mathrm{Ph} \mathrm{CH}), 130.69(\mathrm{Ph} \mathrm{CH}), 133.57(\mathrm{Ph}$ $\mathrm{CH}), 142.82$ (triazole $\mathrm{C}$ or $\mathrm{Ph} \mathrm{C}$ ), 145.68 (triazole $\mathrm{C}$ or $\mathrm{Ph} \mathrm{C}$ ), $166.59(\mathrm{C}=\mathrm{O})$ ). HRMS (ESI) calcld for $\mathrm{C}_{22} \mathrm{H}_{29} \mathrm{~N}_{4} \mathrm{O}_{14} \mathrm{~S}^{-}: 605.140646$. Found: 605.138433 .

\section{Methyl 2',3',4'-tri-O-acetyl-6-[4-(\{[4-(aminosulfonyl)benzoyl]oxy\} methyl)-1H-triazol-1-} yl]-6-deoxy- $\beta$-D-glucopyranoside (3g). The title compound was prepared according to General Procedure 1 and isolated as white foam (123 mg, $0.21 \mathrm{mmol}, 89 \%) . R_{f} 0.42(8: 2$ EtOAc:Hexanes). ${ }^{1} \mathrm{H}$ NMR (400 MHz, DMSO-d $\left.)_{6}\right): \delta 1.92\left(\mathrm{OAc} \mathrm{CH}_{3}\right), 1.96\left(\mathrm{OAc} \mathrm{CH}_{3}\right), 1.99$ $\left(\mathrm{OAc} \mathrm{CH}_{3}\right), 2.99\left(\mathrm{OAc} \mathrm{CH}_{3}\right), 4.13\left(\mathrm{ddd},{ }^{3} J_{5^{\prime}-4^{\prime}}=10.8 \mathrm{~Hz},{ }^{3} J_{5^{\prime}-6},=8.4 \mathrm{~Hz},{ }^{3} J_{5^{\prime}-6^{\prime}},=2.8 \mathrm{~Hz}, 1 \mathrm{H}\right.$, $\left.\mathrm{H}_{5}\right), 4.52\left(\mathrm{dd},{ }^{2} J_{6-6},=14.4 \mathrm{~Hz},{ }^{3} J_{6-5}=8.4 \mathrm{~Hz}, 1 \mathrm{H}, \mathrm{H}_{6}\right), 4.62\left(\mathrm{dd},{ }^{2} J_{6^{\prime}}{ }^{-6} 6^{\prime}=14.4 \mathrm{~Hz},{ }^{3} J_{6^{\prime}}{ }_{-5}=3.0\right.$ $\left.\mathrm{Hz}, 1 \mathrm{H}, \mathrm{H}_{6^{\prime}}\right), 4.80-4.84\left(\mathrm{~m}, 2 \mathrm{H}, \mathrm{H}_{1}, \mathrm{H}_{2}{ }^{\prime}\right), 4.87-4.91\left(\mathrm{~m}, 1 \mathrm{H}, \mathrm{H}_{4}{ }^{\prime}\right), 5.22-5.23(\mathrm{~m}, 1 \mathrm{H}$, $\mathrm{H}_{3}$ ), 5.41 (s, 2H, $\mathrm{CH}_{2} \mathrm{O}$ ), 7.53 (br s, $2 \mathrm{H}, \mathrm{SO}_{2} \mathrm{NH}_{2}$ ), $7.91-8.08$ (m, 4H, Ph $\mathrm{CH}$ ), 8.27 (s, triazole $\mathrm{H}) ;{ }^{13} \mathrm{C}\left\{{ }^{1} \mathrm{H}\right\}$ NMR $\left(100 \mathrm{MHz}, \mathrm{DMSO}-d_{6}\right): \delta 20.99\left(\mathrm{OAc}_{\mathrm{CH}}\right), 21.05\left(\mathrm{OAc}_{3}\right)$, $21.17\left(\mathrm{OAc} \mathrm{CH}_{3}\right), 50.59\left(\mathrm{C}_{6}{ }^{\prime}\right), 55.17\left(\mathrm{OCH}_{3}\right), 59.06\left(\mathrm{CH}_{2} \mathrm{O}\right), 68.03\left(\mathrm{C}_{5}\right), 70.08\left(\mathrm{C}_{1}\right.$, or $\left.\mathrm{C}_{2}{ }^{\prime}\right)$, $70.20\left(\mathrm{C}_{1}\right.$, or $\left.\mathrm{C}_{2}\right), 70.35\left(\mathrm{C}_{3}{ }^{\prime}\right), 96.57\left(\mathrm{C}_{1},\right), 126.55($ triazole $\mathrm{CH}), 126.77(\mathrm{Ph} \mathrm{CH}), 130.58(\mathrm{Ph}$ $\mathrm{CH}), 132.75(\mathrm{Ph} \mathrm{C}), 142.34$ (triazole $\mathrm{C}$ or $\mathrm{Ph} \mathrm{C}), 148.87$ (triazole $\mathrm{C}$ or $\mathrm{Ph} \mathrm{C}), 165.11(\mathrm{C}=\mathrm{O})$, $170.03(\mathrm{C}=\mathrm{O}), 170.27(\mathrm{C}=\mathrm{O}), 170.34(\mathrm{C}=\mathrm{O})$. HRMS (ESI) calcd for $\mathrm{C}_{23} \mathrm{H}_{27} \mathrm{~N}_{4} \mathrm{O}_{12} \mathrm{~S}^{-}$: 
583.135167. Found: 583.133906. Anal $\left(\mathrm{C}_{23} \mathrm{H}_{28} \mathrm{~N}_{4} \mathrm{O}_{12} \mathrm{~S} \bullet 0.5 \mathrm{H}_{2} \mathrm{O}\right) \mathrm{C}, \mathrm{H}$; N: calcd, 9.44; found, 8.04; S: calcd, 5.40; found, 4.95.

\section{Methyl 6-deoxy-6-(4-\{4-Sulfamoylbenzoyloxy\}methyl-1H-1,2,3-triazol-1-yl)- $\alpha-D-$}

glucopyranoside (39'). The title compound was prepared according to procedure 1 and isolated as white solid (75 mg, $0.16 \mathrm{mmol}, 72 \%$ ). $R_{f} 0.52$ (3:7 EtOH:CHCl 3 ); mp $121-122$ ${ }^{\circ} \mathrm{C} .{ }^{1} \mathrm{H}$ NMR $\left(400 \mathrm{MHz}, 2 \% \mathrm{D}_{2} \mathrm{O}\right.$ in DMSO-d 6 ): $\delta 2.85\left(\mathrm{~s}, 3 \mathrm{H}, \mathrm{OCH}_{3}\right), 2.98\left(\mathrm{dd},{ }^{3} J_{4}{ }^{\prime}-5,=10.0\right.$ $\left.\mathrm{Hz},{ }^{3} J_{4^{\prime}-3^{\prime}}=8.8 \mathrm{~Hz}, 1 \mathrm{H}, \mathrm{H}_{4^{\prime}}\right), 3.16\left(\mathrm{dd}^{3}{ }^{3} \mathrm{~J}_{2^{\prime}-3^{\prime}}=9.6 \mathrm{~Hz},{ }^{3} \mathrm{~J}_{2^{\prime}-1},=3.6 \mathrm{~Hz}, 1 \mathrm{H}, \mathrm{H}_{2}{ }^{\prime}\right), 3.32-3.37$ $\left(\mathrm{m}, 1 \mathrm{H}, \mathrm{H}_{3^{\prime}}\right), 3.61-3.66\left(\mathrm{~m}, 1 \mathrm{H}, \mathrm{H}_{5^{\prime}}\right), 4.38\left(\mathrm{dd},{ }^{2} J_{6^{\prime}-6^{\prime}},=14.0,{ }^{3} J_{6^{\prime}-5^{\prime}}=9.2 \mathrm{~Hz}, 1 \mathrm{H}, \mathrm{H}_{6^{\prime}}\right), 4.43$

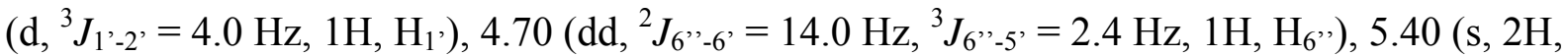
$\left.\mathrm{CH}_{2} \mathrm{O}\right), 7.90-8.07(\mathrm{~m}, 4 \mathrm{H}, \mathrm{Ph}), 8.16(\mathrm{~s}, 1 \mathrm{H}$, triazole $\mathrm{CH}) ;{ }^{13} \mathrm{C}\left\{{ }^{1} \mathrm{H}\right\}$ NMR $(100 \mathrm{MHz}, 2 \%$ $\mathrm{D}_{2} \mathrm{O}$ in DMSO-d $\left.d_{6}\right): \delta 51.01\left(\mathrm{C}_{6}\right), 54.04\left(\mathrm{OCH}_{3}\right), 58.39\left(\mathrm{CH}_{2} \mathrm{O}\right), 70.41\left(\mathrm{C}_{5}\right), 71.66\left(\mathrm{C}_{4}, \mathrm{C}_{2}\right)$, $73.06\left(\mathrm{C}_{3}\right), 99.67\left(\mathrm{C}_{1}{ }^{\prime}\right), 125.89($ triazole $\mathrm{CH}), 126.08(\mathrm{Ph} \mathrm{CH}), 129.89(\mathrm{Ph} \mathrm{CH}), 132.08(\mathrm{Ph}$ C), 141.41 (triazole $\mathrm{C}$ or $\mathrm{Ph} \mathrm{C}$ ), 148.75 (triazole $\mathrm{C}$ or $\mathrm{Ph} \mathrm{C}), 164.43(\mathrm{C}=\mathrm{O})$ ). HRMS (ESI) calcd for $\mathrm{C}_{17} \mathrm{H}_{21} \mathrm{~N}_{4} \mathrm{O}_{9} \mathrm{~S}^{-}: 457.10347$. Found: 457.103411 .

CA inhibition assay. An Applied Photophysics stopped-flow instrument has been used for assaying the $\mathrm{CA}$ catalysed $\mathrm{CO}_{2}$ hydration activity. ${ }^{23}$ Phenol red (at a concentration of $0.2 \mathrm{mM}$ ) has been used as indicator, working at the absorbance maximum of $557 \mathrm{~nm}$, with $10 \mathrm{mM}$ Hepes ( $\mathrm{pH} 7.5$ ) as buffer, $0.1 \mathrm{M} \mathrm{Na}_{2} \mathrm{SO}_{4}$ (for maintaining constant the ionic strength), at 25 ${ }^{\circ} \mathrm{C}$, following the $\mathrm{CA}$-catalyzed $\mathrm{CO}_{2}$ hydration reaction for a period of $10-100 \mathrm{~s}$ (the uncatalyzed reaction needs around $60-100 \mathrm{~s}$ in the assay conditions, whereas the catalyzed ones are of around 6-10 s). The $\mathrm{CO}_{2}$ concentrations ranged from 1.7 to $17 \mathrm{mM}$ for the determination of kinetic parameters. For each inhibitor, tested in the concentration range between $0.01 \mathrm{nM}$ to $100 \mu \mathrm{M}$, at least six traces of the initial $5-10 \%$ of the reaction have been 
used for determining the initial velocity. The uncatalyzed rates were determined in the same manner and subtracted from the total observed rates. Stock solutions of inhibitor $(1 \mathrm{mM})$ were prepared in distilled-deionized water with $10-20 \%(\mathrm{v} / \mathrm{v})$ DMSO (which is not inhibitory at these concentrations) and dilutions up to $0.01 \mathrm{nM}$ were done thereafter with distilleddeionized water. Inhibitor and enzyme solutions were preincubated together for $15 \mathrm{~min}$ at room temperature prior to assay, in order to allow for the formation of the E-I complex. The inhibition constants were obtained by non-linear least-squares methods using PRISM 3. The curve-fitting algorithm allowed us to obtain the IC50 values, working at the lowest concentration of substrate of $1.7 \mathrm{mM}$ ), from which $K_{\mathrm{i}}$ values were calculated by using the Cheng-Prusoff equation. ${ }^{24-28}$ The catalytic activity (in the absence of inhibitors) of these enzymes was calculated from Lineweaver-Burk plots, as reported earlier, and represent the mean from at least three different determinations. ${ }^{24-28}$ Enzyme concentrations in the assay system were: $9.2 \mathrm{nM}$ for hCA I, $7.3 \mathrm{nM}$ for hCA II and $8.5 \mathrm{nM}$ for hCA IX. Enzymes used here were recombinant ones, prepared and purified as described earlier. ${ }^{24-28,30}$

\section{Acknowledgement}

This work was financed in part by the Australian Research Council (DP0343419); the Eskitis Institute for Cell and Molecular Therapies and the School of Science (Griffith University) and an EU grant of the $6^{\text {th }}$ framework programme (EUROXY project) (University of Florence).

Supporting Information Available: Elemental analysis data and ${ }^{1} \mathrm{H}$ NMR spectra for compounds. This material is available free of charge via the Internet at http://pubs.acs.org. 


\section{References}

1. Supuran, C.T. Carbonic anhydrases: Catalytic and inhibition mechanism, distribution and physiological roles. In Carbonic Anhydrase: Its Inhibitors and Activators; Supuran, C.T., Scozzafava, A., Conway, J., Eds.; CRC Press: Florida, 2004; pp 1-24.

2. Scozzafava, A.; Supuran, C.T. Carbonic anhydrase inhibitors. Curr. Med. Chem. Imm., Endoc. \& Metab. Agents. 2001, 1, 61-97.

3. Supuran, C.T.; Scozzafava, A.; Casini, A. Carbonic anhydrase inibitors. Med. Res. Rev. 2003, 23, 146-189.

4. Pastorekova, S.; Parkkila, S.; Pastorek, J.; Supuran, C.T. Carbonic anhydrases: Current state of the art, therapeutic applications and future prospects. J. Enz. Inhib. Med. Chem. 2004, 19, 199-229.

5. Scozzafava, A.; Owa, T.; Mastrolorenzo, A.; Supuran, C.T. Anticancer and antiviral sulfonamides. Curr. Med. Chem. 2003, 10, 925-953.

6. Pastorekova, S.; Casini, A; Scozzafava, A.; Vullo, D.; Pastorek, J.; Supuran, C.T. Carbonic anhydrase inhibitors: The first selective, membrane-impermeant inhibitors targeting the tumor-associated isozyme IX. Bioorg. Med. Chem. Lett. 2004, 14, 869873.

7. Pastorekova, S.; Pastorek, J. Cancer-related carbonic anhydrase isozymes. In Carbonic anhydrase - Its inhibitors and activators; Supuran, C.T., Scozzafava, A., Conway, J., Eds.; CRC Press: Florida, USA, 2004; pp. 253-280.

8. Erlanson, D.A.; McDowell, R.S.; O’Brien, T. Fragment-based drug discovery. J. Med. Chem. 2004, 47, 3463.

9. Lipinski, C.A.; Lombardo, F.; Dominy, B.W.; Feeney, P.J. Experimental and computational approaches to estimate solubility and permeability in drug discovery and development settings. Adv. Drug Del. Rev. 2001, 46, 3-26. 
10. ten Tije A.J.; Verweij J.; Loos W.J.; Sparreboom A. Pharmacological effects of formulation vehicles. Implications for cancer chemotherapy. Clin. Pharmacokinet. 2003, 42, 665-685.

11. Gradishar, W.J.; Tjulandin, S.; Davidson, N.; Shaw, H.; Desai, N.; Bhar, P.; Hawkins, M.; O'Shaughnessy, J. Phase III trial of nanoparticle albumin-bound paclitaxel compared with polyethylated castor oil-based paclitaxel in women with breast cancer. J. Clin. Oncol. 2005, 23, 7794-7803.

12. Blanchfield, J.; Toth, I. Lipid, sugar and liposaccharide based delivery systems 2. Curr. Med. Chem. 2004, 11, 1241-1253.

13. Drinnan, N.B.; Vari, F. Aspects of the stability and bioavailability of carbohydrates and carbohydrate derivatives. Mini Rev. Med. Chem. 2003, 3, 633-649.

14. Macmillan, D.; Daines A.M. Recent developments in the synthesis and discovery of oligosaccharides and glycoconjugates for the treatment of disease. Curr. Med. Chem. 2003, 10, 2733-2773.

15. Koreeda, M.; Houston, T.A.; Shull, B.K.; Klemke, E.; Tuinman, R.J. Iodine catalyzed Ferrier reaction 1. A mild and highly versatile glycosylation of hydroxy and phenolic groups. Synlett. 1995, 90-92.

16. Service, R. F.; Science 2001, 291, 2342-2342.

17. Winum, J-,V.; Casini, A.; Mincione, F.; Starnotti, M.; Montero, J.-L.; Scozzafava, A.; Supuran, C.T. Carbonic anhydrase inhibitors: $N$-(p-sulfamoylphenyl)- $\alpha-D-$ glycopyranosylamines as topically acting antiglaucoma agents in hypertensive rabbits. Bioorg. Med. Chem. Lett. 2004, 14, 225-229.

18. Wilkinson, B.L.; Bornaghi, L.F.; Poulsen, S.-A.; Houston, T.A. Synthetic utility of glycosyl triazoles in carbohydrate chemistry. Tetrahedron 2006, 62, 8115-8125. 
19. Mincione, F.; Starnotti, M.; Menabuoni, L.; Scozzafava, A.; Casini, A.; Supuran, C.T. Carbonic anhydrase inhibitors: 4-sulfamoyl-benzenecarboxamides and 4-chloro-3sulfamoyl-benzenecarboxamides with strong topical antiglaucoma properties. Bioorg. Med. Chem. Lett. 2001, 11, 1787-1791.

20. Kunz, H.; Waldmann, H. Construction of disaccharide $N$-glycopeptides - Synthesis of the linkage region of the transmembrane-neuraminidase of an influenza virus. Angew. Chem. Int. Ed. 1985, 24, 883-885.

21. Cottaz, S.; Brimacombe, J.S.; Ferguson, M.A.J. An imino-linked carba-disaccharide a-D-mannosidase inhibitor. Carb. Res. 1993, 247, 341 - 345.

22. Rostovtsev, V.V.; Green, L.G.; Fokin, V.V.; Sharpless, K.B. A stepwise Huisgen cycloaddition process: Copper(I)-catalyzed regioselective "ligation" of azides and terminal alkynes. Angew. Chem. Int. Ed. 2002, 41, 2596-2599.

23. Khalifah, R.G. The carbon dioxide hydration activity of carbonic anhydrase. J. Biol. Chem. 1971, 246, 2561-2573.

24. Winum, J.-Y.; Vullo, D.; Casini, A.; Montero, J.-L.; Scozzafava, A.; Supuran, C.T. Carbonic anhydrase inhibitors: Inhibition of transmembrane, tumor-associated isozyme IX, and cytosolic isozymes I and II with aliphatic sulfamates. J. Med. Chem, 2003, 46, 5471-5477.

25. Svastova, E.; Hulikova, A.; Rafajova, M.; Zatovicova, M.; Gibadulinova, A.; Casini, A.; Cecchi, A.; Scozzafava, A.; Supuran, C.T.; Pastorek, J.; Pastorekova, S. Hypoxia activates the capacity of tumor-associated carbonic anhydrase IX to acidify extracellular pH. FEBS Letters 2004, 577, 439-445.

26. Winum, J.-Y.; Vullo, D.; Casini, A.; Montero, J.-L.; Scozzafava, A.; Supuran, C.T. Carbonic anhydrase inhibitors. Inhibition of cytosolic isozymes I and II and 
transmembrane, tumor-associated isozyme IX with sulfamates including EMATE also acting as steroid sulfatase inhibitors. J. Med. Chem. 2003, 46, 2197-2204.

27. Cecchi, A.; Hulikova, A.; Pastorek, J.; Pastorekova, S.; Scozzafava, A.; Winum, J.-Y.; Montero, J.-L.; Supuran, C.T. Carbonic anhydrase inhibitors. Sulfonamides inhibit isozyme IX mediated acidification of hypoxic tumors. Fluorescent sulfonamides design as probes of membrane-bound carbonic anhydrase isozymes involvement in tumorigenesis. J. Med. Chem. 2005, 48, 4834-484.

28. Casey, J. R.; Morgan, P. E.; Vullo, D.; Scozzafava, A.; Mastrolorenzo, A.; Supuran, C. T. Carbonic anhydrase inhibitors. Design of selective, membrane-impermeant inhibitors targeting the human tumor-associated isozyme IX. J. Med. Chem. 2004, 47, $2337-2347$.

29. Hang, H.C.; Bertozzi, C.R. Chemoselective approaches to glycoprotein assembly. Acc. Chem. Res. 2001, 34, 727-736.

30. Vullo, D.; Franchi, M.; Gallori, E.; Pastorek, J.; Scozzafava, A.; Pastorekova, S.; Supuran, C.T. Carbonic anhydrase inhibitors. Inhibition of the tumor-associated isozyme IX with aromatic and heterocyclic sulfonamides. Bioorg. Med. Chem. Lett. 2003, 13, 1005-1009. 
Table 1. Inhibition and selectivity ratio data for sulfonamides $\mathbf{2}, \mathbf{3}$, the 28 new glycoconjugates and standard inhibitors against human isozymes hCA I, II, and IX.

\begin{tabular}{|c|c|c|c|c|c|}
\hline \multirow[b]{2}{*}{ Compound } & \multicolumn{3}{|c|}{$K_{\mathbf{i}}(\mathbf{n M})^{a}$} & \multicolumn{2}{|c|}{ Selectivity ratios ${ }^{b}$} \\
\hline & $\mathrm{hCA} \mathrm{I}$ & $\mathrm{hCA} \mathrm{II}{ }^{c}$ & $\mathrm{hCA} \mathrm{IX}^{d}$ & $\begin{array}{r}K_{\mathrm{i}}(\mathrm{hCA} \text { I) } / \\
(\mathrm{hCA} \mathrm{IX)}\end{array}$ & $\begin{array}{r}K_{\mathrm{i}}(\mathrm{hCA} \text { II) } \\
(\mathrm{hCA} \text { IX) }\end{array}$ \\
\hline AZA & 900 & 12 & 25 & 36 & 0.48 \\
\hline MZA & 780 & 14 & 27 & 28.9 & 0.52 \\
\hline EZA & 25 & 8 & 34 & 0.74 & 0.24 \\
\hline BRZ & 15 & 9 & 42 & 0.36 & 0.21 \\
\hline DCP & 1200 & 38 & 50 & 24 & 0.76 \\
\hline IND & 31 & 15 & 24 & 1.30 & 0.63 \\
\hline 2 & 6100 & 47 & 113 & 54.0 & 0.42 \\
\hline 3 & 4000 & 45 & 104 & 38.5 & 0.43 \\
\hline $2 a$ & 5600 & 384 & 430 & 13.0 & 0.89 \\
\hline $2 a^{\prime}$ & 2000 & 8.2 & 442 & 4.5 & 0.02 \\
\hline $3 \mathbf{a}$ & 2300 & 119 & 1238 & 1.86 & 0.10 \\
\hline $3 \mathbf{a}^{\prime}$ & 4400 & 7.0 & 183 & 24.0 & 0.04 \\
\hline $2 b$ & 8700 & 470 & 76 & 114.5 & 6.2 \\
\hline $2 b^{\prime}$ & 6600 & 7.4 & 360 & 18.3 & 0.02 \\
\hline $3 \mathbf{b}$ & 3600 & 6.8 & 132 & 27.3 & 0.05 \\
\hline $3 b^{\prime}$ & 5800 & 8.1 & 65 & 89.2 & 0.12 \\
\hline $2 c$ & 2400 & 279 & 103 & 23.3 & 2.71 \\
\hline $2 c^{\prime}$ & 2700 & 128 & 420 & 6.4 & 0.30 \\
\hline $3 c$ & 4900 & 7.3 & 114 & 43.0 & 0.06 \\
\hline
\end{tabular}




\begin{tabular}{|c|c|c|c|c|c|}
\hline $3 c^{\prime}$ & 7.7 & 5.8 & 96 & 0.08 & 0.06 \\
\hline 2d & 1200 & 45 & 124 & 9.68 & 0.36 \\
\hline 2d' & 9100 & 265 & 238 & 38.2 & 1.11 \\
\hline 3d & 5100 & 218 & 1200 & 4.25 & 0.18 \\
\hline 3d' & 4800 & 7.3 & 108 & 44.4 & 0.07 \\
\hline $2 e$ & 5800 & 7.6 & 471 & 12.3 & 0.02 \\
\hline $2 e^{\prime}$ & 2400 & 378 & 23 & 104.3 & 16.4 \\
\hline $3 e$ & 5400 & 7.0 & 125 & 43.2 & 0.06 \\
\hline $3 e^{\prime}$ & 9.6 & 7.2 & 241 & 0.04 & 0.03 \\
\hline $2 f$ & 9200 & 7.5 & 221 & 41.6 & 0.03 \\
\hline $2 f^{\prime}$ & 9500 & 267 & 1100 & 8.64 & 0.24 \\
\hline $3 f$ & 3900 & 423 & 130 & 30.0 & 3.25 \\
\hline 3f' & 3500 & 7.3 & 39 & 89.7 & 0.19 \\
\hline $2 g$ & 10400 & 44 & 135 & 77.0 & 0.33 \\
\hline $2 g^{\prime}$ & 9300 & 90 & 204 & 45.6 & 0.44 \\
\hline $3 g$ & 3400 & 50 & 54 & 63.0 & 0.93 \\
\hline $3 g^{\prime}$ & 3100 & 8.6 & 67 & 46.3 & 0.13 \\
\hline
\end{tabular}

${ }^{a}$ Errors in the range of $\pm 5-10 \%$ of the reported value, from three determinations. ${ }^{b}$ The $K_{\mathrm{i}}$ ratios are indicative of isozyme selectivity. ${ }^{c}$ Human (cloned) isozymes, by the $\mathrm{CO}_{2}$ hydration method. ${ }^{23-28}$ ${ }^{d}$ Catalytic domain of human (cloned) isozyme, by the $\mathrm{CO}_{2}$ hydration method. ${ }^{23-28}$ 


\section{Figure Legends}

Figure 1. Azido sugar building block panel: $\mathbf{a - g}(\mathrm{R}=\mathrm{Ac})$ and $\mathbf{a}^{\prime}-\mathbf{g}$ ' $(\mathrm{R}=\mathrm{H})$.

Figure 2. Glycoconjugate benzene sulfonamides. 


\section{Scheme Titles and Footnotes}

Scheme 1. $\mathrm{Cu}(\mathrm{I})$ catalyzed 1,3-dipolar cycloaddition reaction (1,3-DCR) of azido sugars and terminal alkynes. ${ }^{18}$

\section{Scheme 2. ${ }^{a}$}

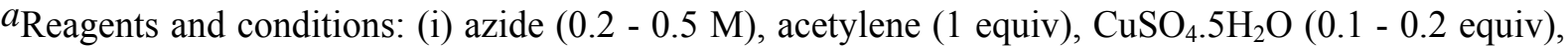
sodium ascorbate $\left(0.2-0.4\right.$ equiv), $1: 1 \mathrm{t}-\mathrm{BuOH} / \mathrm{H}_{2} \mathrm{O}, 40{ }^{\circ} \mathrm{C}, 30 \mathrm{~min}-1 \mathrm{~h}, 58-96 \%$; (ii) $\mathrm{NaOCH}_{3}$, $\mathrm{CH}_{3} \mathrm{OH}, \mathrm{rt}, 2 \mathrm{~h}$, quantitative. 
Figure 1.

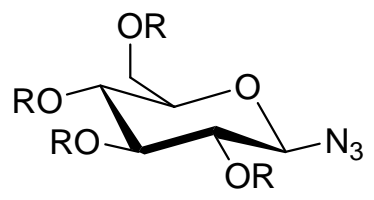

a, $\mathbf{a}^{\prime}$

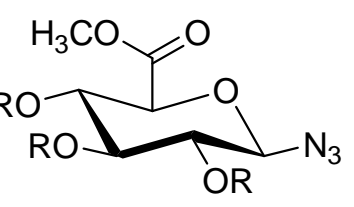

e, $\mathbf{e}^{\prime}$

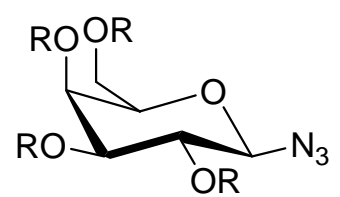

b, b'
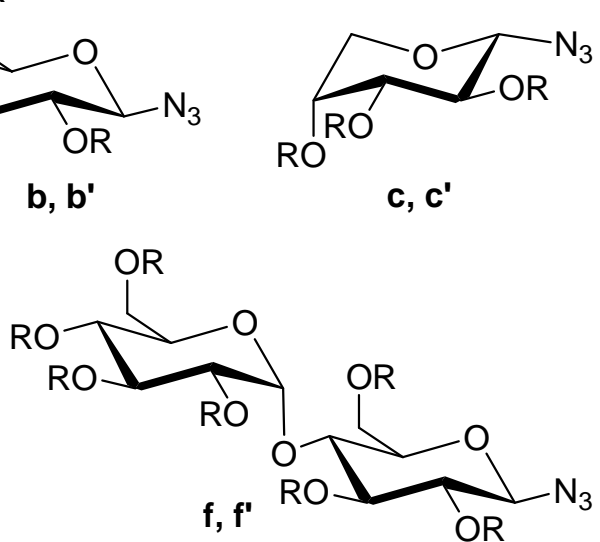

c, $\mathbf{c}^{\prime}$

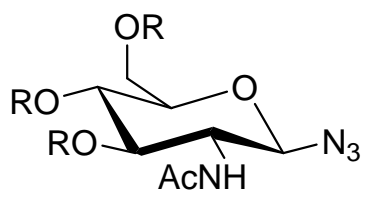

d, d'

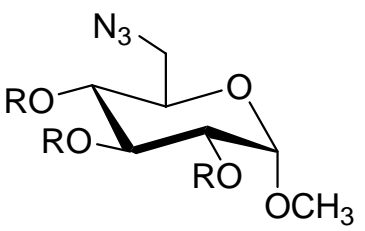

g, g' 
Figure 2.

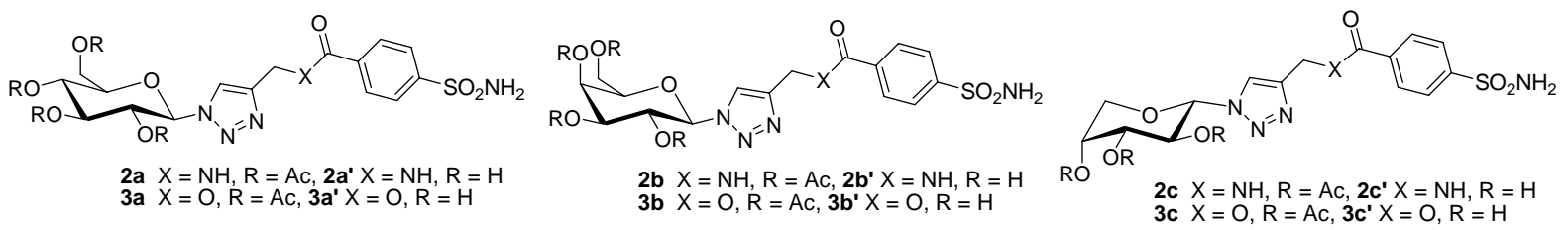

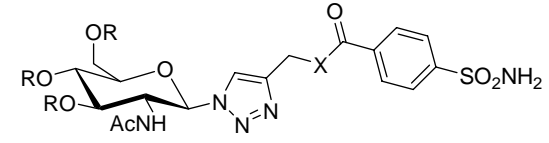

2d $X=N H, R=A c, 2 d^{\prime} X=N H, R=H$
3d $X=O, R=A c, 3 d^{\prime} X=O, R=H$

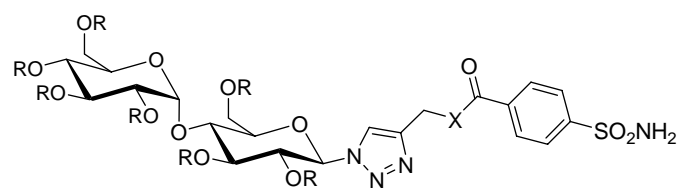

2f $X=N H, R=A c, 2 f^{\prime} X=N H, R=H$ 3f $X=O, R=A c, 3 f^{\prime} X=O, R=H$

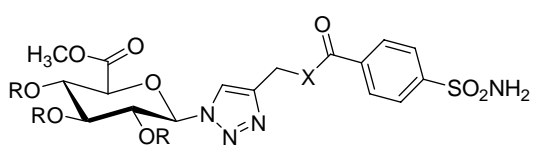

2e $X=N H, R=A c, 2 e^{\prime} X=N H, R=H$ 3e $X=O, R=A c, 3 e^{\prime} X=O, R=H$

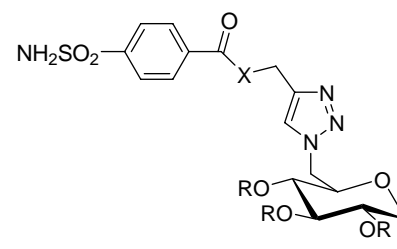

2g $X=N H, R=A c, 2 g^{\prime} X=N H, R=H$ $3 \mathbf{g} X=O, R=A c, \mathbf{s g}^{\prime} X=O, R=H$ 
Scheme 1.

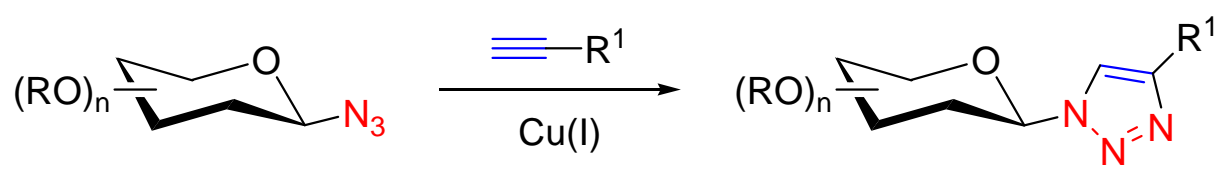


Scheme 2.

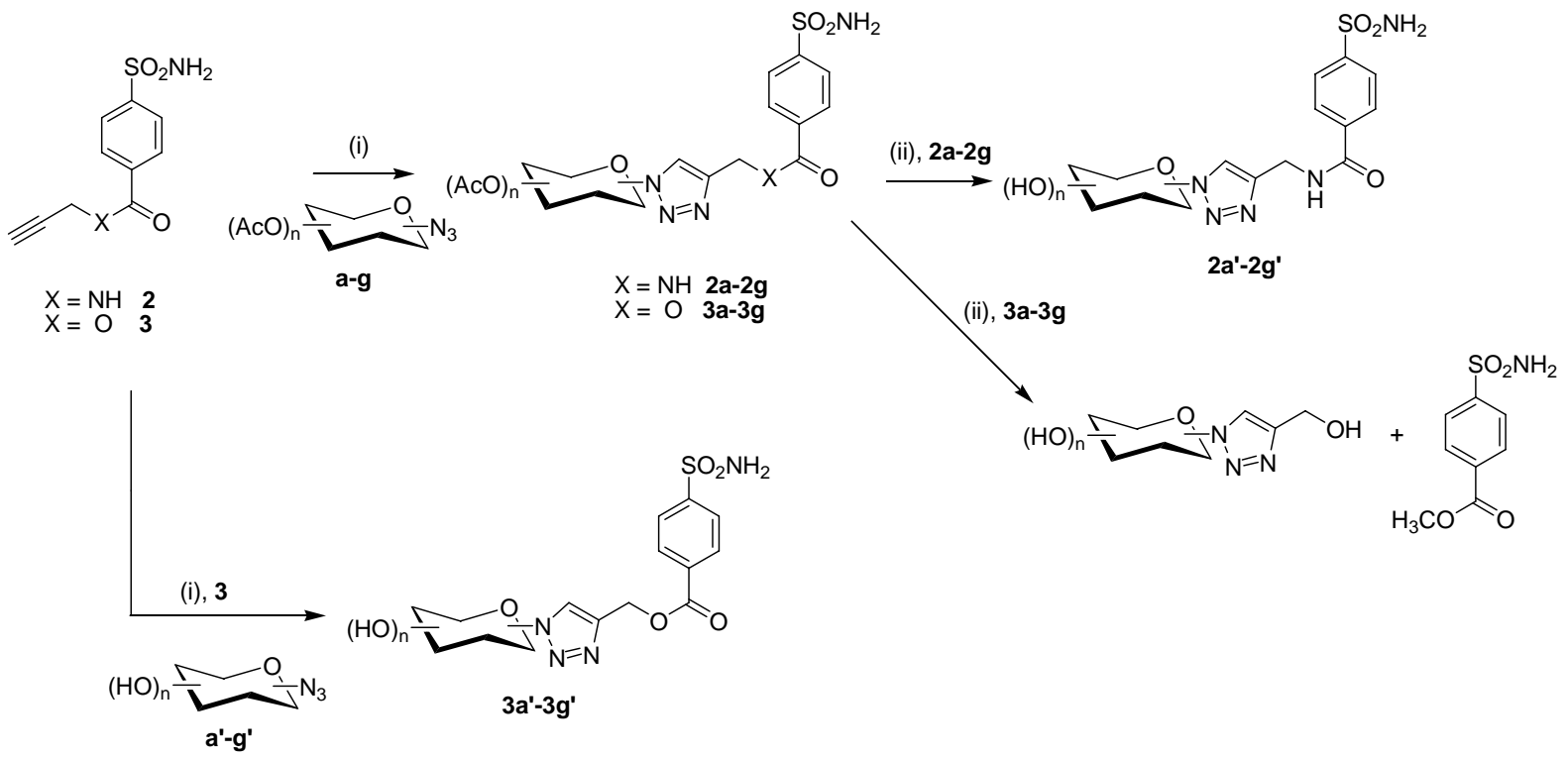




\section{Chemical Structures Group A}<smiles>CC(=O)Nc1nnc(S(N)(=O)=O)s1</smiles>

acetazolamide<smiles>CCN[C@H]1C[C@H](CC)S(=O)(=O)c2sc(S(N)(=O)=O)cc21</smiles>

brinzolamide<smiles>NS(=O)(=O)c1cc(Cl)c(Cl)c(S(N)(=O)=O)c1</smiles>

dichlorphenamide<smiles>CCOc1ccc2nc(S(N)(=O)=O)sc2c1</smiles>

ethoxazolamide<smiles>NS(=O)(=O)c1ccc(S(=O)(=O)Nc2cccc3c(Cl)c[nH]c23)cc1</smiles>

indisulam 
Chemical Structure Group B

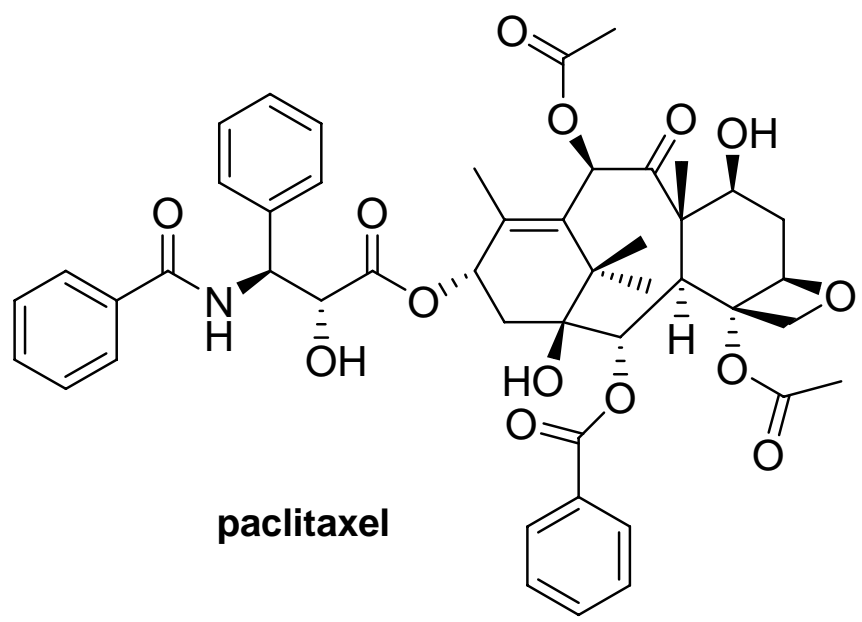


Chemical Structures Group C<smiles>C#CCNC(=O)c1ccc(S(N)(=O)=O)cc1</smiles> 
Table of Contents Graphic

A novel class of carbonic anhydrase inhibitors: glycoconjugate benzene sulfonamides prepared by "click-tailing"

Brendan L. Wilkinson, ${ }^{\dagger}$ Laurent F. Bornaghi ${ }^{\dagger}$ Todd A. Houston, $₫$ Alessio Innocenti, $§$ Claudiu T. Supuran ${ }^{*} \S$ and Sally-Ann Poulsen ${ }^{*, \dagger}$
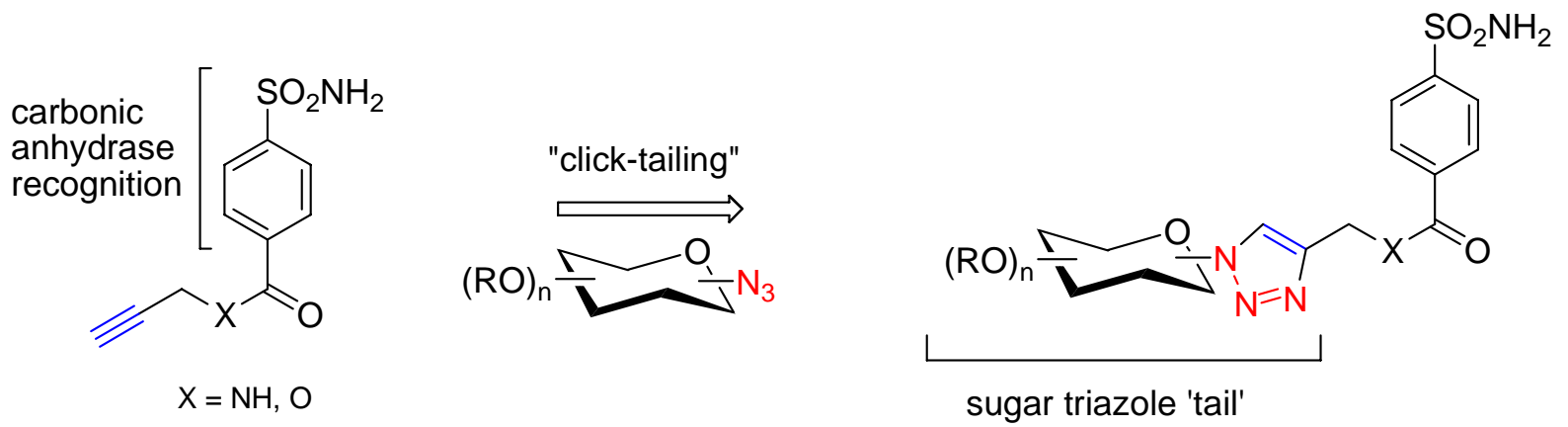